\title{
Hemispheric asymmetry in the human brain and in Parkinson's disease is linked to divergent epigenetic patterns in neurons
}

\author{
Peipei Li ${ }^{1}$, Elizabeth Ensink', Sean Lang ${ }^{1}$, Lee Marshall ${ }^{1}$, Meghan Schilthuis ${ }^{1}$, Jared Lamp ${ }^{2,3}$, Irving Vega ${ }^{2,3}$ and \\ Viviane Labrie ${ }^{1,4^{*}}$ (D)
}

\begin{abstract}
Background: Hemispheric asymmetry in neuronal processes is a fundamental feature of the human brain and drives symptom lateralization in Parkinson's disease (PD), but its molecular determinants are unknown. Here, we identify divergent epigenetic patterns involved in hemispheric asymmetry by profiling DNA methylation in isolated prefrontal cortex neurons from control and PD brain hemispheres. DNA methylation is fine-mapped at enhancers and promoters, genome-wide, by targeted bisulfite sequencing in two independent sample cohorts.

Results: We find that neurons of the human prefrontal cortex exhibit hemispheric differences in DNA methylation. Hemispheric asymmetry in neuronal DNA methylation patterns is largely mediated by differential CpH methylation, and chromatin conformation analysis finds that it targets thousands of genes. With aging, there is a loss of hemispheric asymmetry in neuronal epigenomes, such that hemispheres epigenetically converge in late life. In neurons of PD patients, hemispheric asymmetry in DNA methylation is greater than in controls and involves many PD risk genes. Epigenetic, transcriptomic, and proteomic differences between PD hemispheres correspond to the lateralization of PD symptoms, with abnormalities being most prevalent in the hemisphere matched to side of symptom predominance. Hemispheric asymmetry and symptom lateralization in PD is linked to genes affecting neurodevelopment, immune activation, and synaptic transmission. PD patients with a long disease course have greater hemispheric asymmetry in neuronal epigenomes than those with a short disease course.
\end{abstract}

Conclusions: Hemispheric differences in DNA methylation patterns are prevalent in neurons and may affect the progression and symptoms of PD.

Keywords: Hemisphere asymmetry, Parkinson's disease, Aging, Enhancer, Neurons, Epigenetics

\section{Background}

Parkinson's disease (PD) is a severe, irreversible neurodegenerative disease involving motor symptoms that are unilateral at onset for over $85 \%$ of patients [1-3]. The lateralization of motor symptoms results from an asymmetric pattern of neurodegeneration in the brain [4-8]. PD patients have hemispheric asymmetry in neuronal dysfunction in both the nigrostriatal system and cortical brain structures, such that the hemisphere contralateral

\footnotetext{
* Correspondence: viviane.labrie@vai.org

${ }^{1}$ Center for Neurodegenerative Science, Van Andel Institute, Grand Rapids, Ml 49503, USA

${ }^{4}$ Division of Psychiatry and Behavioral Medicine, College of Human Medicine, Michigan State University, Grand Rapids, MI 49503, USA

Full list of author information is available at the end of the article
}

to PD motor symptom predominance shows greater neuronal and synaptic dysfunction than the ipsilateral side [4-8]. Right/left brain asymmetries in PD appear early, in preclinical stages $[4,8,9]$. Though PD symptoms eventually affect both body sides as the disease progresses, clinical asymmetry remains directionally stable and detectable even at advanced disease stages $[10,11]$. Furthermore, asymmetric motor presentation is linked to the rate of disease progression [12-14]. Cognitive symptoms also differ between subgroups of lateralized PD patients (i.e., visuospatial tasks, language, verbal memory, and psychosis differ between patients with left vs. right motor symptom predominance) [14-19]. Despite the prevalence of asymmetric brain changes in $\mathrm{PD}$

(c) The Author(s). 2020 Open Access This article is distributed under the terms of the Creative Commons Attribution 4.0 International License (http://creativecommons.org/licenses/by/4.0/), which permits unrestricted use, distribution, and reproduction in any medium, provided you give appropriate credit to the original author(s) and the source, provide a link to the Creative Commons license, and indicate if changes were made. The Creative Commons Public Domain Dedication waiver (http://creativecommons.org/publicdomain/zero/1.0/) applies to the data made available in this article, unless otherwise stated. 
and its relevance to disease progression and clinical manifestations, the factors rendering neurons more vulnerable to degeneration in one hemisphere over the other are unknown.

Epigenetic regulation represents a mechanism through which genetic, environmental, and aging risk factors could plausibly trigger hemispheric differences in neuronal and synaptic loss. Epigenetic marks like DNA methylation enable dynamic regulation of gene expression throughout the life of a neuron $[20,21]$. In the brain, divergent DNA methylation signatures facilitate the functional specialization of neurons and brain subregions [22-24]. During early development, hemispheric asymmetry in DNA methylation is linked to the lateralization of nervous system organization, which affects hemisphere dominance for handedness, cognitive processes, and language [25-27]. Furthermore, DNA methylation status governs the activity of gene regulatory elements such as enhancers and promoters, which affect the establishment of left-right asymmetries in various tissues [28-30]. Hence, epigenetic variation may influence asymmetrical gene expression patterns in the brain, which if pathogenic could contribute to PD.

In post-mitotic neurons, disruption of DNA methylation induces lasting changes in synaptic architecture and cellular signaling that can promote neurodegenerative processes [31-33]. Genome-wide studies have identified abnormalities in DNA methylation in the PD brain [3437]. In addition, several studies of the PD brain have demonstrated a loss of DNA methylation at the $\alpha$ synuclein gene promoter, which may contribute to elevated $\alpha$-synuclein expression; a major PD risk factor and a main component of Lewy pathology in this disease [38-41]. Furthermore, aging is the strongest risk factor for $\mathrm{PD}$, and epigenetic changes contribute to aging processes [20, 42, 43]. Genes linked to neurodegeneration exhibit epigenetic changes with age, and accelerated epigenetic aging is observed in PD [44, 45]. A hemispheric imbalance in the accumulation of DNA methylation abnormalities affecting genes involved in disease pathophysiology may explain the lateralization of clinical symptoms in PD, though this has yet to be examined.

Here, we identify hemispheric asymmetry in neuronal DNA methylation patterns in the control and PD brain. DNA methylation was fine-mapped at gene regulatory elements, enhancers, and promoters, genome-wide, in isolated neurons of the prefrontal cortex of PD patients and controls. In two independent cohorts, we find that neurons of PD patients have extensive hemispheric asymmetry in DNA methylation, exceeding that of control individuals. In particular, regulatory elements of PD risk genes (identified in genetic studies) show prominent epigenetic asymmetry in PD patients. Inter-hemispheric differences in the epigenome of PD patients closely associates with symptom lateralization, such that epigenetic changes are most apparent in the hemisphere matched to the predominant side of symptom presentation. Furthermore, hemispheric asymmetry in DNA methylation patterns is linked to genes with transcriptomic and proteomic differences between hemispheres. In aging, there is a progressive loss of hemispheric asymmetry in the epigenomes of the control and PD brain. For PD patients, epigenetic asymmetry between hemispheres is associated with differences in disease progression, as PD patients with a long disease course have more hemispheric asymmetry. Together, our results support that neurons of PD patients have pronounced hemispheric differences in DNA methylation that are associated with the lateralization of PD symptoms.

\section{Results}

\section{Hemispheric asymmetry in DNA methylation patterns in} neurons

To determine whether there are hemispheric differences in DNA methylation that could impact neuronal functions in the healthy and PD brain, we comprehensively fine-mapped DNA methylation in neurons isolated from either the left or right prefrontal cortex of PD patients and controls $(n=57$ and 48 individuals, respectively; Additional file 2). Neuronal nuclei from hemispheres were isolated by an established antibody- and flow cytometry-based approach [32, 46] (Additional file 1: Figure S1). DNA methylation was profiled at all brain enhancers and promoters across the genome, including active and poised/bivalent elements, as defined by the NIH Roadmap Epigenomics Project (ChromHMM 18state model). Genome-wide mapping of DNA methylation at enhancers and promoters was performed with a targeted bisulfite sequencing strategy, known as the bisulfite padlock probe approach. The padlock probe library consisted of 59,009 probes targeting 35,288 regulatory elements (Additional file 3 ). In PD and control neurons, we investigated a total of 633,803 modified cytosines, of which 106,047 were CpG and 527,756 were $\mathrm{CpH}$ sites (Additional file 1: Figure S2).

We examined whether there were left-right hemispheric differences in the epigenome of control neurons, and whether hemispheric differences were greater in PD neurons than in control neurons. Our analysis examined individual cytosine sites (CpGs and $\mathrm{CpHs}$ ) for hemispheric asymmetry in control and PD neurons, and adjusted for age, sex, post-mortem interval, and neuronal subtype proportion. Neuronal subtype proportion refers to the proportion of glutamatergic to GABAergic neurons, as determined by neuronal subtype deconvolution. The PD prefrontal cortex did not exhibit a hemispherespecific loss in subtypes of glutamatergic and GABAergic neurons (Additional file 4). Also, inter-sample 
correlations for $\mathrm{CpG}$ and $\mathrm{CpH}$ were similar (Additional file 1: Table S1).

Cortical neurons of the human brain exhibited a prominent hemispheric asymmetry in DNA methylation ( $n=25$ left and 23 right hemispheres of controls; Fig. 1a). Inter-hemispheric differences in DNA methylation occurred at 2587 cytosine sites at enhancers and promoters in control prefrontal cortex neurons $(q<0.05$, robust linear regression with contrasts; Fig. 1a; Additional file 5). In particular, the left hemisphere had higher DNA methylation levels than the right hemisphere $(82.7 \%$ of significant cytosines had more DNA methylation in the left hemisphere; $p<10^{-15}$, Fisher's exact test; Fig. 1b), which was largely due to differential $\mathrm{CpH}$ methylation (Fig. 1b, c; Additional file 1: Figure S3). Indeed, $\mathrm{CpH}$ methylation, an epigenetic mark correlating with repression of enhancers and promoters in neurons [21], was the primary epigenetic contributor to hemispheric asymmetry (2371 CpHs and $216 \mathrm{CpGs}, p<10^{-34}$ for $\mathrm{CpH}$ enrichment, hypergeometric test; Fig. 1d). In neurons, the hemispheric asymmetry of DNA methylation most often affected cis-acting regulatory elements, located proximal to transcription start sites (peak within $5 \mathrm{~kb}$ and average distance $47.2 \pm 1.4 \mathrm{~kb}$ from transcription start sites; average DNA methylation change $6.09 \% \pm 0.39 \%$ at CpGs and $1.34 \% \pm 0.05 \%$ at $\mathrm{CpHs}$; Fig. 1c; Additional file 1: Figure S4 and S5).

We then determined the gene targets of the enhancers and promoters that showed hemispheric asymmetry in their epigenome. We used promoter-centric chromatin interactions identified in human cortical neurons and excitatory neurons $[47,48]$. We found 1247 genes targeted by enhancers and promoters with hemispheric asymmetry. To further capture proximal interactions, we used an in silico cis-regulatory element prediction tool [49]. In total, we found 3068 genes having hemispheric differences in the epigenetic regulation of their cisregulatory elements (Additional file 6). Pathway analysis revealed that genes with hemispheric asymmetry in epigenetic regulation were primarily involved in neurodevelopment and brain diseases, including neurodegenerative diseases $(q<0.05$, hypergeometric test; Fig. 1e). These findings demonstrate that hemispheric asymmetry in neuronal epigenomes is prevalent in the human brain and may lead to inter-hemispheric differences in vulnerability to brain illnesses.

We next determined that PD patient neurons have substantially more hemispheric asymmetry in DNA methylation than control neurons $(n=23$ PD-left hemisphere, 34 PD-right, 25 control-left, 23 control-right). There were 6207 cytosine sites exhibiting left vs. right hemispheric asymmetry in DNA methylation at enhancers and promoters $(q<0.05$, robust linear regression with contrasts; Fig. 2a; Additional file 5), of which 3894 sites had greater asymmetry in PD patients $(62.7 \% ; p<$ $10^{-15}$, Fisher's exact test; Fig. $2 \mathrm{~b}$ ). $\mathrm{CpH}$ sites were an important source of the hemispheric differences in DNA methylation $(5465 \mathrm{CpHs}, 0.82 \% \pm 0.02 \%$ change in $\mathrm{PD}$; 742 CpGs, $4.40 \% \pm 0.14 \%$ change in PD; $p<10^{-25}$ for $\mathrm{CpH}$ enrichment, hypergeometric test; Fig. 2c; Additional file 1: Figure S3). In PD, hemispheric asymmetry in DNA methylation most often affected cis-acting regulatory elements, located near transcription start sites (peak within $5 \mathrm{~kb}$ and on average within $45.9 \pm 1.0 \mathrm{~kb}$ of transcription start sites). We then identified the gene targets of the enhancers and promoters, using the chromatin conformation data from human cortical neurons and excitatory neurons $[47,48]$ in combination with the in silico cis-regulatory element prediction tool, as described above. We found that PD patients had 4691 genes with abnormal hemispheric asymmetry in epigenetic regulation, relative to controls (Additional file 6). Moreover, DNA methylation abnormalities across PD hemispheres affected many PD risk genes [50] (Fig. 2a). Thus, the neurons of PD patients exhibit extensive hemispheric asymmetry in the epigenetic regulation of genes, including known PD risk genes.

\section{PD symptom lateralization involves epigenetic and transcriptional divergence between hemispheres}

We examined whether DNA methylation abnormalities in PD were most apparent in the hemisphere matched to the side of clinical motor symptom predominance. Our highly characterized PD cases had information about the side of the body on which symptoms predominated (Additional file 2). We profiled neurons of $17 \mathrm{PD}$ patients with the hemisphere matched to the symptomdominant side (the contralateral hemisphere) and 20 unmatched (the ipsilateral hemisphere), and we compared these PD groups to 48 control subjects. Neurons from the hemisphere matched to symptom dominance exhibited more DNA methylation differences relative to control hemispheres than did neurons from the unmatched hemisphere (Fig. 2d). There were 3587 DNA methylation sites differing between the matched hemisphere of PD patients and controls, compared to 2283 between the unmatched hemisphere of PD patients and controls $(q<$ 0.05 , robust linear regression with contrasts, adjusted for age, sex, postmortem interval, brain hemisphere (left or right), and neuronal subtype proportion; Additional file 5). The identification of gene targets (as described above) revealed that differentially methylated regulatory elements in PD neurons affected 3431 genes in the hemisphere matched to symptom predominance and 2722 genes in the unmatched hemisphere, of which 1697 genes were altered in both hemispheres (Fig. 2d; Additional file 6). Overall, there were substantially more genes altered in the symptom-dominant hemisphere: 


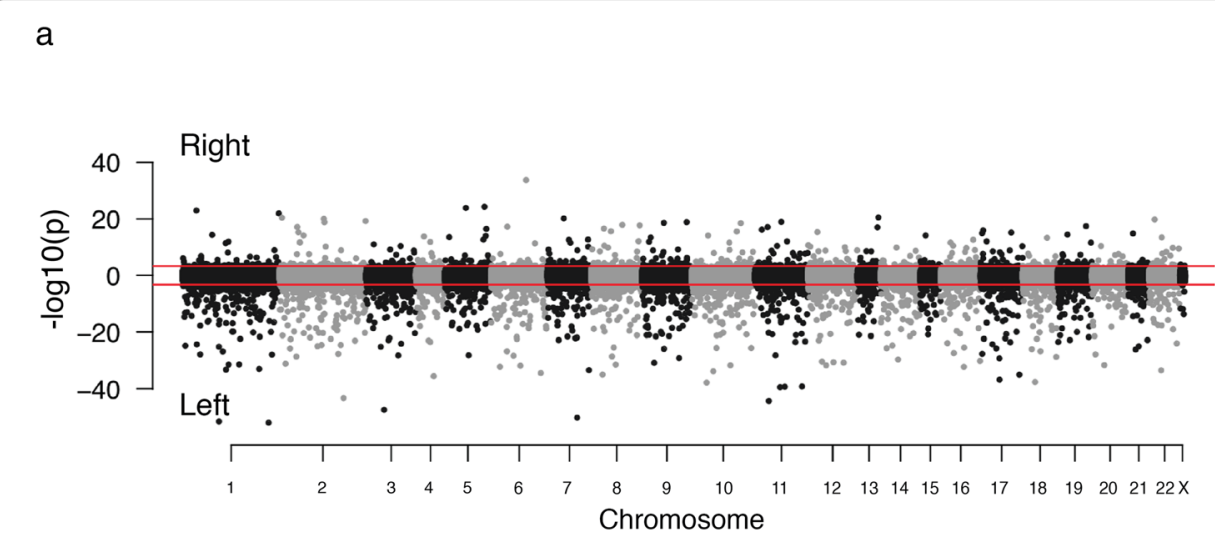

b Increased DNA methylation

Right

$p<10^{-15}$

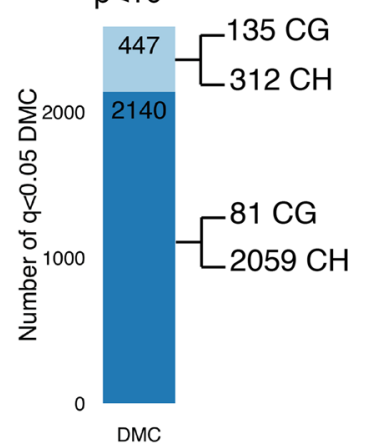

C

e

Biological processes nervous system development neurogenesis neuron differentiation generation of neurons anatomical structure development neuron development multicellular organanismal process cell development
cellopment neuron projection development cell morphogenesis involved in neuron differentiation regulation of multicellular organismal process developmental process cell projection morphogenesis plasma membrane neuron projection morphogenesis regulation of developmental process cell part morphogenesis cell morphogenesis $L$

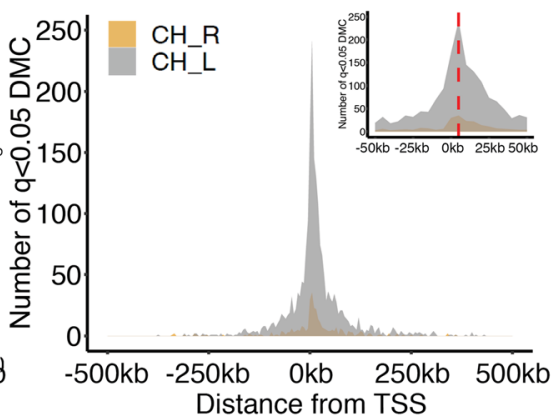

Distance from TSS

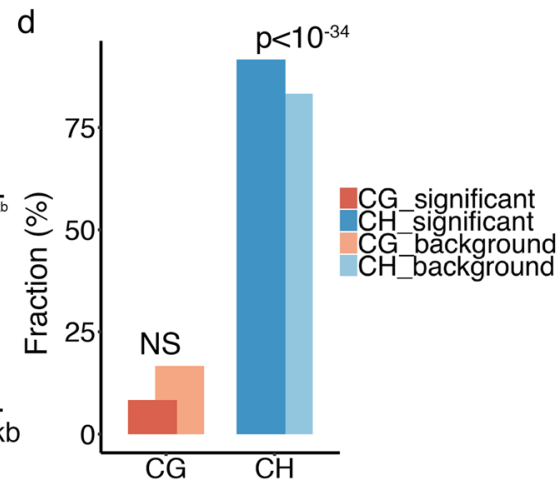

Diseases

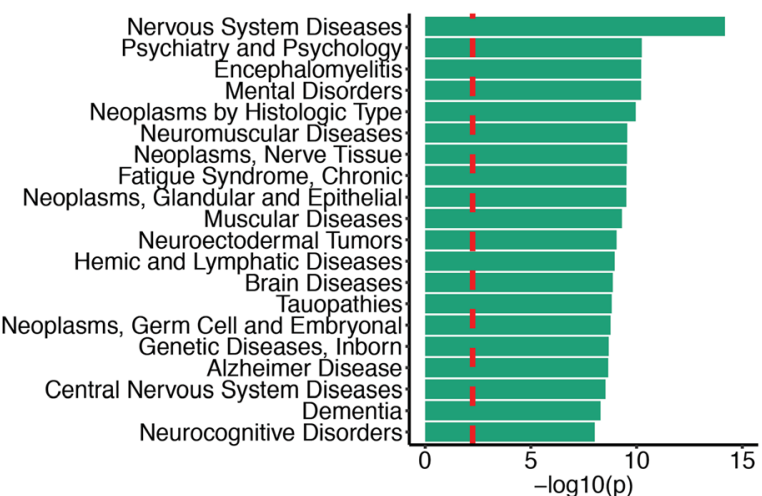

Fig. 1 (See legend on next page.) 
(See figure on previous page.)

Fig. 1 Hemispheric asymmetry in DNA methylation in cortical neurons of the human brain. a Manhattan plot showing DNA methylation differences between neurons of the left and right hemisphere of the prefrontal cortex of control individuals, after adjusting for age, sex, postmortem interval, and neuronal subtype proportion. DNA methylation was mapped at enhancers and promoters of neurons from the control prefrontal cortex ( $n=25$ left hemispheres, 23 right). $-\log 10(p)$ refers to the significance of differentially methylated cytosines, with the sign corresponding to the hemisphere side exhibiting higher DNA methylation levels. Threshold for genome-wide significance (red line) is $q<0.05$, as determined by robust linear regression with contrasts. $\mathbf{b}$ Bar plot showing the distribution of differentially methylated cytosines (DMC) across hemispheres. The number of significant cytosine sites that show greater DNA methylation levels in the left or right hemisphere is shown. The $p$ value represents the enrichment of cytosines with increased DNA methylation in the left hemisphere, by Fisher's exact test. CpG and CpH site contribution to differential methylation in each hemisphere is shown. c Genomic location of DNA methylation changes involved in hemispheric asymmetry in human cortical neurons. The location of $\mathrm{CpG}$ (left panel) and $\mathrm{CpH}$ (right panel) sites with increased DNA methylation in the left or right hemisphere is shown. Inset is a close-up of the transcription start site region. $\mathbf{d} \mathrm{CpG}$ and $\mathrm{CpH}$ involvement in inter-hemispheric DNA methylation differences. The percent number of significantly altered $\mathrm{CpG}$ or $\mathrm{CpH}$ sites differing between hemispheres (relative to background) is shown. The enrichment of cytosine context involved in hemispheric asymmetry was determined by hypergeometric test. e Top biological processes and disease pathways of genes affected by hemispheric asymmetry in DNA methylation. Pathway analysis was done by MetaCore. Threshold for significance (red dashed line) is $q<0.05$

3404 genes had more DNA methylation abnormalities at their regulatory elements in the PD hemisphere matched to symptom dominance than in the unmatched hemisphere $(q<0.05$ cytosines with fold change in matched vs. control > unmatched vs. control; Additional file 6). Thus, prominent hemispheric asymmetry in the epigenomes of PD patient neurons mirrors the lateralization of clinical symptoms.

We determined whether epigenetic divergence between hemispheres is relevant to asymmetry in transcriptional patterns. We performed a transcriptomic analysis of the prefrontal cortex from control hemispheres and from PD hemispheres matched or unmatched to the symptom-dominant side $(n=12$ controls, 13 PD-matched, 11 PD-unmatched; Fig. 2d). As in our epigenetic analysis, we found that the hemisphere matched to the symptom-dominant side had greater transcriptional differences relative to control hemispheres than did the unmatched hemisphere (Fig. 2d). Specifically, the matched PD hemisphere had 1440 differentially expressed genes, while the unmatched PD hemisphere had 827 differentially expressed genes, compared to controls $(q<0.1$, generalized linear regression with contrasts; Fig. 2d; Additional file 7), after adjusting for age, sex, brain hemisphere, neuron proportion, RIN, and other sources of variation (by RUVSeq [51]). There were 1121 genes with greater transcriptional changes in the matched PD hemisphere than in the unmatched hemisphere. There was also a significant association between changes in DNA methylation at gene regulatory elements and changes in corresponding transcript levels in PD $(p<0.001$, interaction term in linear regression; Additional file 1: Figure S6). Thus, hemispheric differences in the epigenome are accompanied by functionally relevant transcriptomic alterations.

We then determined convergent pathways affected by epigenetic and transcriptional changes involved in hemispheric asymmetry in PD. We identified pathways altered in PD hemispheres matched or unmatched to the side of symptom predominance relative to controls, and then compared these pathways between the PD hemispheres. The PD hemisphere matched to symptom predominance had prominent changes in antigen presentation and immune activation, neuronal development, DNA damage and cell cycle, extracellular matrix, and synaptic transmission $(q<0.05$, g:Profiler and GSEA; Fig. 2e). Hence, asymmetry in PD may result from brain hemisphere differences in immune responses, neurodevelopment, and neurotransmission.

\section{Validation of hemispheric asymmetry in the epigenome with an independent PD and control cohort}

To confirm our discovery that hemispheric asymmetry in DNA methylation in PD exceeds that of controls and corresponds to clinical motor symptom predominance, we replicated our findings with an independent cohort. In this replication study, we examined neurons from both the left and right hemisphere of the same person. DNA methylation was profiled in neuronal nuclei isolated from the prefrontal cortex of 31 controls and 26 PD patients: 12 PD patients with left-side symptom predominance and 14 with right-side predominance (Additional file 2). Fine-mapping of DNA methylation at all brain enhancers and promoters was performed using the same bisulfite padlock probe approach as in our discovery cohort. After data preprocessing, DNA methylation was examined at 815,367 cytosine sites (133,736 CpGs and 681,631 CpHs; Additional file 1: Figure S2). As above, we analyzed hemispheric asymmetry at individual cytosine sites (CpGs and $\mathrm{CpHs}$ ) in neurons of the left and right hemisphere of PD patients and controls, adjusting for age, sex, postmortem interval, and neuronal subtype proportion.

In this independent cohort, we confirmed that hemispheric asymmetry is prevalent in neurons of the human brain. In healthy control neurons, there were 3246 DNA methylation sites with significant differences across hemispheres $(q<0.05$, robust linear regression with 


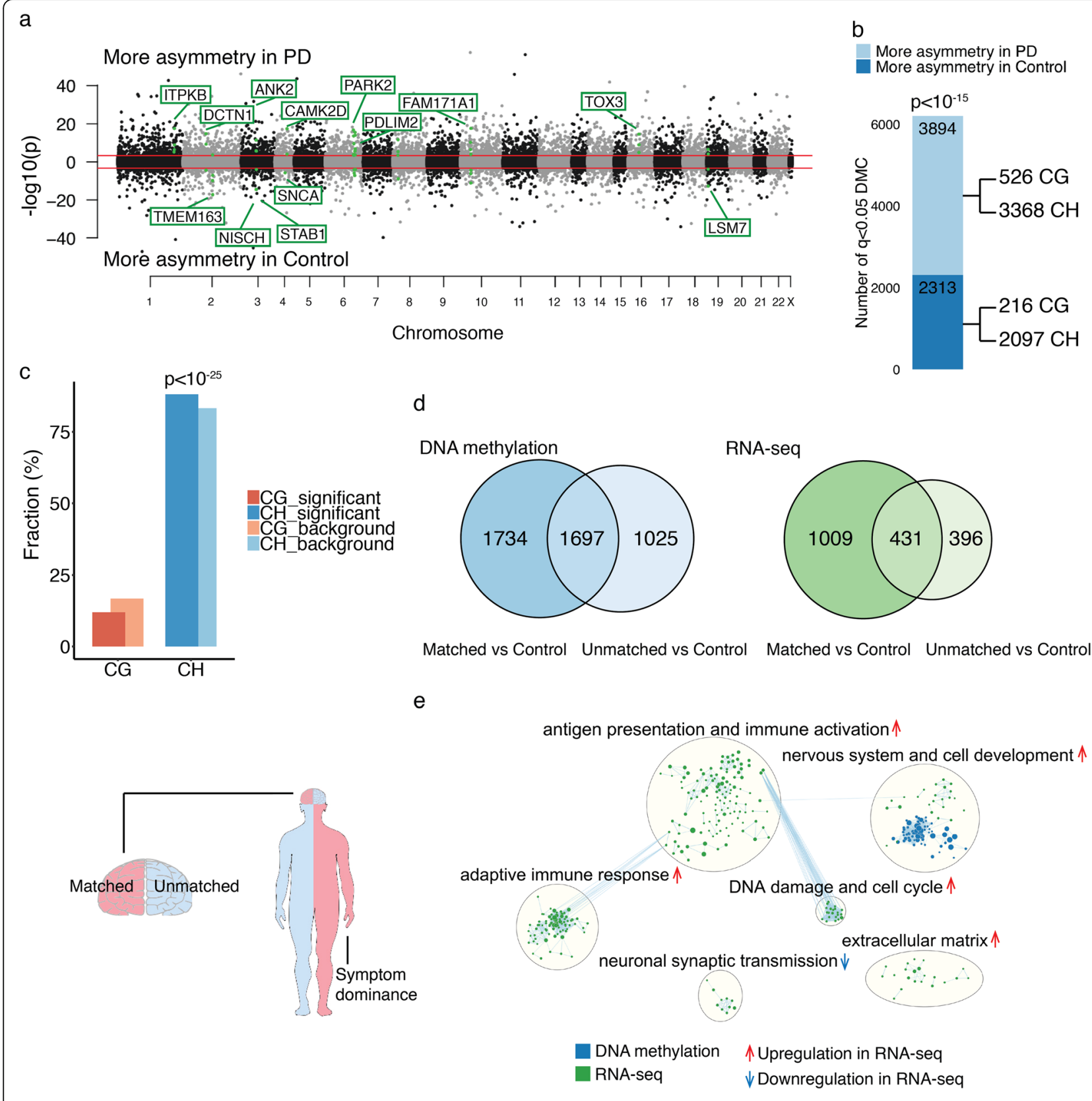

Fig. 2 (See legend on next page.) 


\section{(See figure on previous page.)}

Fig. 2 Epigenetic differences across hemispheres are increased in PD and are relevant to the lateralization of clinical symptoms. a Manhattan plot comparing the hemispheric asymmetry in DNA methylation in neurons of PD patients and controls, after adjusting for age, sex, postmortem interval, and neuronal subtype proportion. DNA methylation differences were profiled at enhancers and promoters in prefrontal cortex neurons of 57 PD patients and 48 controls. $-\log 10(p)$ refers to the significance of differentially methylated cytosines (DMC), with the sign corresponding to the diagnosis group with greater hemispheric asymmetry. Threshold for genome-wide significance (red line) is $q<0.05$. Highlighted are genes implicated in PD risk (familial and/or identified by GWAS [50]) that have enhancers or promoters with 3 or more cytosines exhibiting hemispheric asymmetry in PD. $\mathbf{b}$ Comparison of the degree of hemispheric asymmetry in DNA methylation between PD patients and controls. The number of significant cytosine sites exhibiting more hemispheric asymmetry in PD or control neurons is shown. The $p$ value represents the enrichment of cytosines with greater hemispheric asymmetry in PD relative to controls, by Fisher's exact test. $\mathrm{CpG}$ and $\mathrm{CpH}$ contributions to hemispheric asymmetry are shown. c CpG and $\mathrm{CpH}$ involvement in hemispheric asymmetry changes in PD. The percent number of $\mathrm{CpG}$ or $\mathrm{CpH}$ sites significantly involved in hemispheric asymmetry changes in PD (relative to background) is shown. The enrichment of cytosine context was determined by hypergeometric test. $\mathbf{d}$ DNA methylation and transcriptional differences relevant to the lateralization of PD symptoms. Venn diagram showing the number of genes affected by differential methylation (left panel) or exhibiting differential expression (right panel) in the symptom-dominant (matched) or non-dominant (unmatched) PD hemisphere, relative to controls (DNA methylation: $n=17$ PD-matched, 20 PDunmatched, 48 controls; RNA-seq: $n=13$ PD-matched, 11 PD-unmatched, 12 controls). e Pathway analysis of genes epigenetically and transcriptionally altered in neurons of the PD hemisphere matched or unmatched to side of symptom predominance, relative to control neurons. Only pathway clusters with nodes having greater changes in the hemisphere matched to PD symptom dominance are shown. Pathway enrichment analysis of DNA methylation data was done by g:Profiler (blue nodes) and of RNA-seq data was done by GSEA preranked (green nodes). Epigenetically and transcriptionally dysregulated pathways in PD hemispheres were merged in Enrichment Map using $q<0.05$ pathways. Pathways up- or downregulated in PD hemispheres depicted by red or blue arrows, respectively, as determined by GSEA

contrasts; Additional file 1: Figure S7; Additional files 5 and 6). DNA methylation levels were higher in neurons of the left hemisphere $\left(p<10^{-15}\right.$, Fisher's exact test), and $\mathrm{CpH}$ methylation was again the primary contributor to hemispheric asymmetry in DNA methylation (2898 $\mathrm{CpHs}$ and $348 \mathrm{CpGs} ; p<10^{-19}$ for $\mathrm{CpH}$ enrichment, hypergeometric test; Additional file 1: Figure S7).

Moreover, we replicated in the independent cohort that there is more hemispheric asymmetry in neurons of PD patients than in controls $(q<0.05$, robust linear regression with contrasts; Additional file 1: Figure S8b; Additional files 5 and 6). DNA methylation abnormalities in PD also prevailed on the hemisphere side matched to symptom predominance (Fig. 3a). The symptom-dominant hemisphere had 589 differentially methylated cytosines, while the non-dominant hemisphere had 240 differentially methylated cytosines, relative to controls $(q<0.05$, robust linear regression with contrasts; Additional file 5). Gene targets of enhancers and promoters with hemispheric differences in DNA methylation in the replication cohort were identified as above. We found a total of 991 genes exhibiting more DNA methylation alterations in the symptomdominant PD hemisphere (relative to controls) than in the non-dominant hemisphere (relative to controls) (Additional file 1: Figure S8c; Additional file 6). Moreover, there was a strong overlap between the discovery and replication cohort in the genes with DNA methylation abnormalities preferentially occurring in the symptom-dominant hemisphere $\left(p<10^{-88}\right.$, hypergeometric test; Fig. 3b). This signifies an independent replication of epigenetically dysregulated genes involved in the lateralization of PD symptoms.
We sought to identify the genes that were most strongly associated with asymmetry in PD symptoms. We used the epigenetic and transcriptomic data from our discovery cohort and the epigenetic data from our replication cohort, and searched for the genes most dysregulated in the hemisphere corresponding to the side of symptom dominance. Each gene was ranked according to the significance of change and consistency across the epigenetic and transcriptomic datasets. We identified 34 genes showing consistent preferential dysregulation in the hemisphere matched to symptom predominance (Fig. 3c, d; Additional file 8). In particular, the neuronal cell adhesion molecule 1 (NCAM1), which regulates neuronal development, synaptogenesis, cell-cell interactions, and synaptic plasticity, was central to the abnormalities in the symptom-dominant PD hemisphere (Fig. 3d).

\section{Proteomic analysis of hemispheric asymmetry in PD}

To further explore the genes involved in hemispheric asymmetry in PD, we performed a quantitative proteomic analysis of the PD prefrontal cortex. We first identified 1063 proteins with altered abundances in the prefrontal cortex of PD patients relative to controls $(n=3 \mathrm{PD}$ patients and 3 controls; Additional file 9). We then determined 668 protein differences between the PD hemispheres matched and unmatched to symptom dominance ( $n=5$ PD-matched and 5 PD-unmatched). These analyses were merged to identify 345 disease-relevant proteins that exhibit hemispheric asymmetry in the PD brain (Additional files 9 and 10). Notably, we identified that SNCA ( $\alpha$-synuclein) and NCAM1 were altered in PD and exhibited hemispheric asymmetry (Additional file 1: 


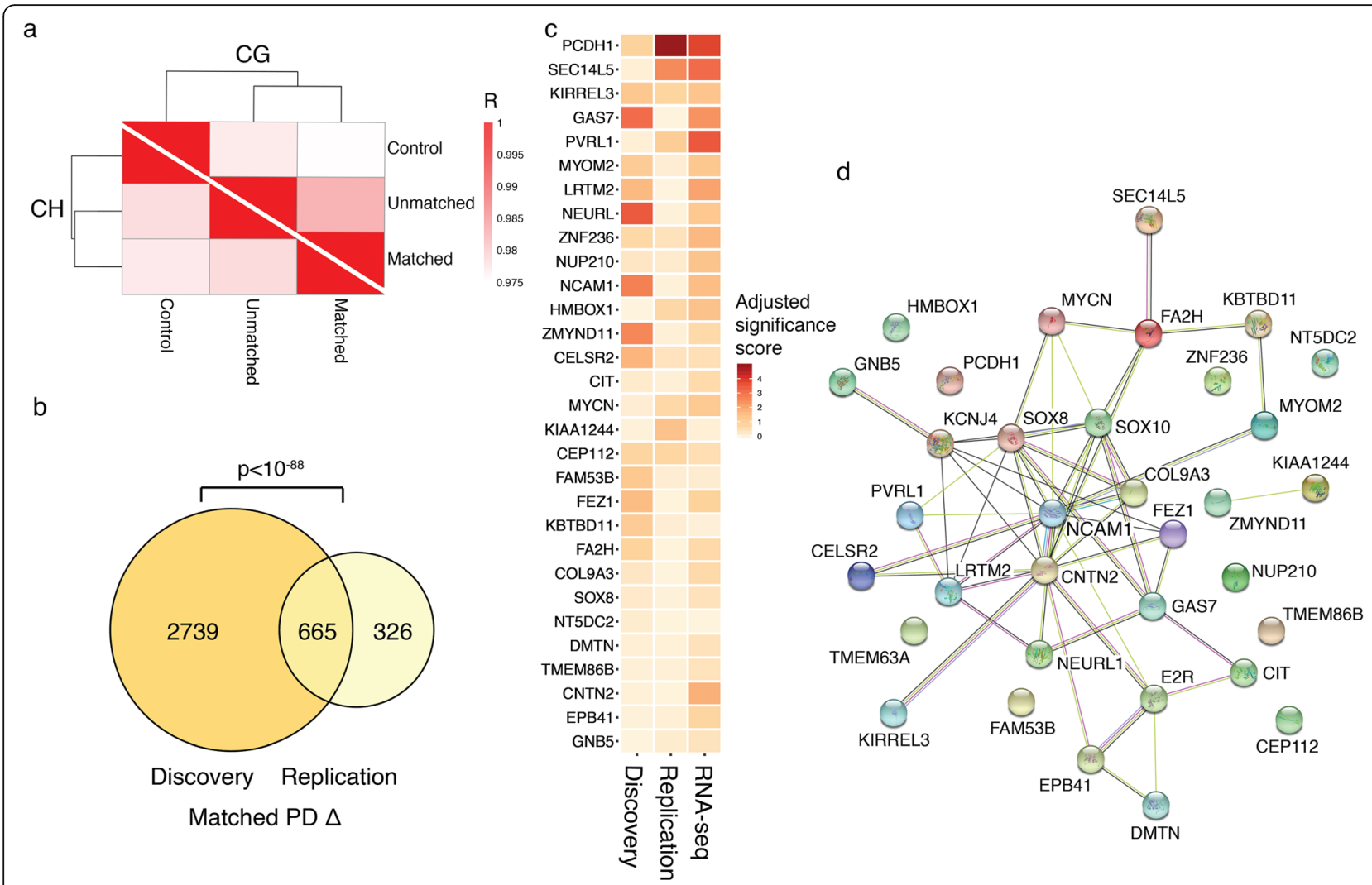

e neuron differentiation and

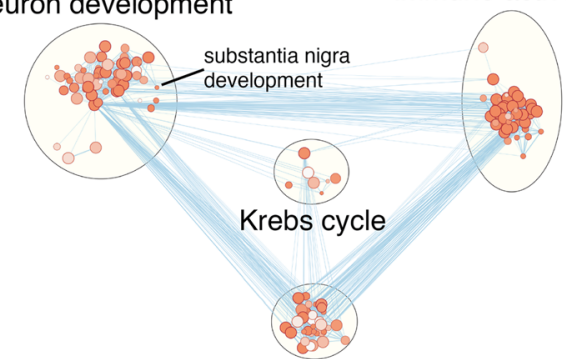

antigen presentation and immune activation

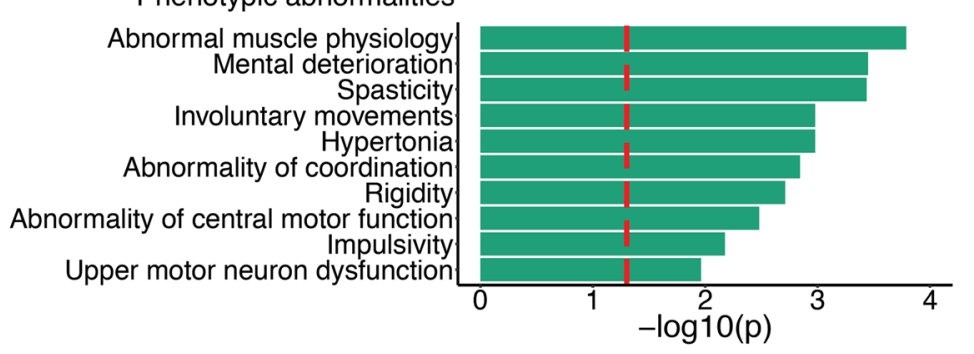

protein targeting to membrane

Fig. 3 Independent validation that epigenetic dysregulation is greater in the symptom-dominant PD hemisphere. DNA methylation at enhancers and promoters was examined in an independent cohort of PD patients and controls. Prefrontal cortex neurons from both hemispheres were examined ( $n=31$ controls, 26 PD patients). a Divergence of epigenetic profiles in neurons of PD hemispheres matched or unmatched to the side of symptom dominance from those of control neurons. Shown are Pearson correlations comparing DNA methylation status of control, matched, and unmatched groups. Darker red signifies a higher DNA methylation similarity between diagnosis groups. $\mathbf{b}$ Concordance between discovery and replication cohorts. Venn diagram showing overlap of discovery and replication cohorts for genes with greater epigenetic abnormalities in the symptom-dominant hemisphere of PD patients. The $p$ value represents the significance of the discovery and replication cohort overlap, by hypergeometric test. c Top 30 genes most associated with PD symptom lateralization. Genes were ranked across datasets to determine the most robustly dysregulated genes in the symptom-dominant hemisphere of PD patients. The discovery and replication DNA methylation data, as well as the RNA-seq data, was used. Genes are listed in ranked order, and the heatmap depicts the adjusted significance score in each dataset. $\mathbf{d}$ Network analysis of top 30 genes involved in symptomatic asymmetry in PD centers on NCAM1. Network analysis performed by STRING. e Pathways of proteomic alterations involved in hemispheric asymmetry and symptom lateralization in PD. Protein changes were determined by mass spectrometry in the prefrontal cortex of PD patients, relative to controls, and between the PD symptom-dominant and non-dominant hemispheres ( $n=3-5$ individuals). Pathway enrichment analysis performed by g:Profiler (nodes are $q<0.05$ pathways, hypergeometric test) and pathways were merged in Enrichment Map. $\mathbf{f}$ Top disease pathways of proteins involved in hemispheric asymmetry in PD. Pathway analysis was done by g:Profiler. Threshold for significance (red dashed line) is $q<0.05$ 
Figure S9). SNCA and NCAM1 levels were highest in the PD hemisphere matched to symptom dominance (Additional file 1: Figure S9; Additional file 9). Pathway analysis of the PD-relevant proteins exhibiting hemispheric asymmetry revealed differences in nervous system development (including substantia nigra development), antigen presentation and immune activation, and protein transport $(q<$ 0.05 , hypergeometric test; Fig. 3e). Moreover, phenotypic abnormalities associated with the protein changes involved in hemispheric asymmetry in PD were related to motor dysfunction, including many hallmark symptoms of PD ( 9 of the top 10 human disease pathways involved in motor dysfunction; $q<0.05$, hypergeometric test; Fig. 3f).
Therefore, proteomic analysis supports the epigenetic and transcriptomic findings that symptom asymmetry in PD is associated with differences in neurodevelopmental processes and immune responses between hemispheres.

\section{Changes in hemispheric asymmetry in neurons with aging} In $\mathrm{PD}$, highly lateralized motor symptoms gradually become more bilateral with increased age and disease duration, though lateralization persists even in advanced PD stages $[10,11]$. We found that hemispheric asymmetry in the epigenome of prefrontal cortex neurons changed with aging, especially for PD patients (Fig. 4). We found 5925 methylated cytosines at enhancers that showed

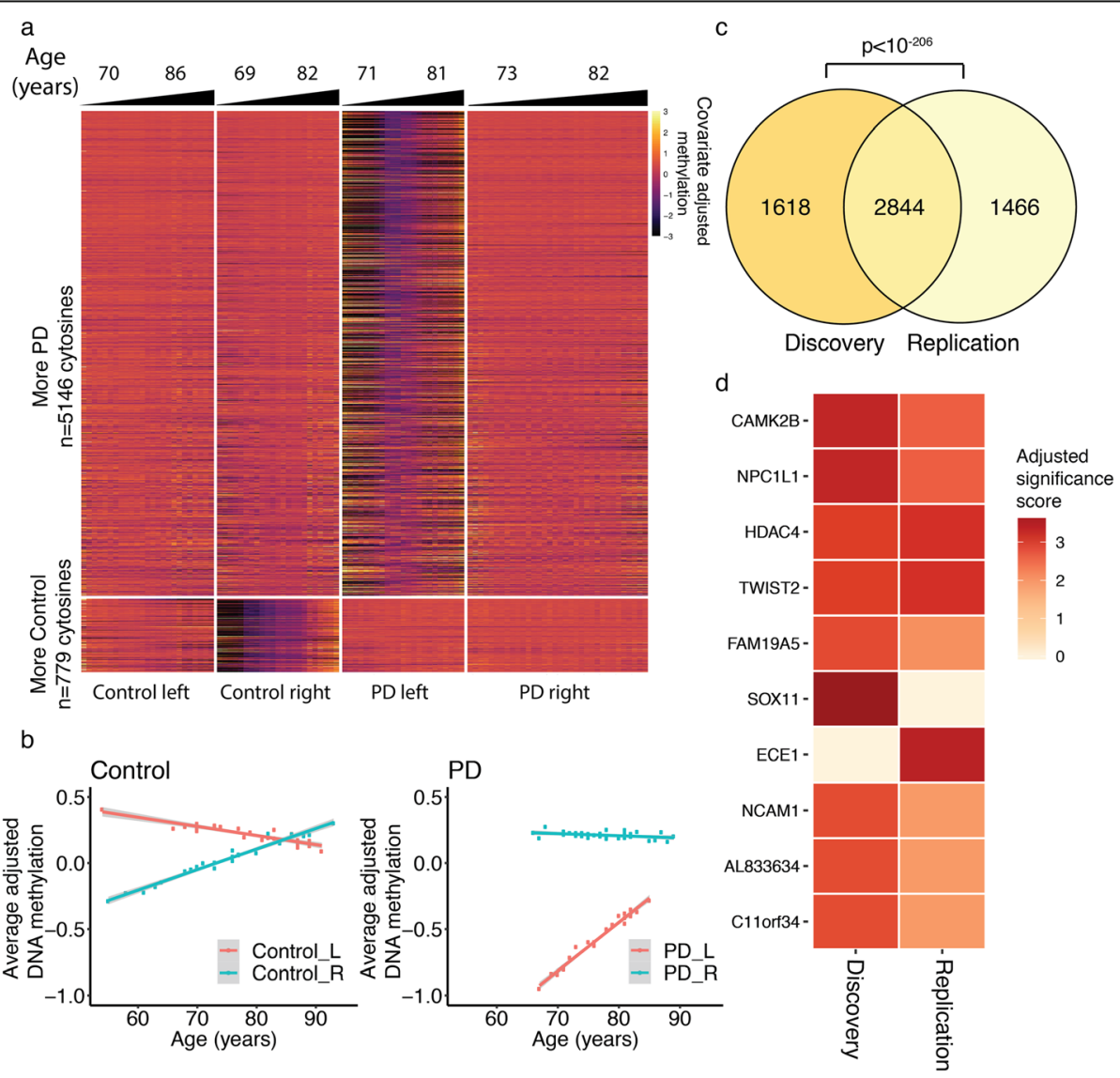

Fig. 4 Progressive loss of hemispheric asymmetry in DNA methylation with aging. Age-dependent changes in DNA methylation in neurons of the left and right hemisphere of PD patients and controls ( $n=23$ PD-left, 34 PD-right, 25 control-left, 23 control-right). a Heatmap showing adjusted DNA methylation levels at the 5925 cytosine sites exhibiting significant changes in hemispheric asymmetry with aging ( $q<0.05$, robust linear model followed by contrasts; DNA methylation adjusted for sex, postmortem interval and neuronal subtype proportion). Cytosine sites with more aging changes in hemispheric asymmetry in PD (upper panels) or in controls (lower panels) are shown. The first and third quartiles of age for each group are shown. $\mathbf{b}$ Scatter plot of aging changes in DNA methylation across hemispheres of controls and PD patients. Averaged adjusted DNA methylation values is shown for the sites significantly associated with aging (DNA methylation adjusted for sex, postmortem interval and neuronal subtype proportion). Data for the left (red) and right (blue) hemisphere is shown for controls (left panel) and PD patients (right panel). The gray area represents confidence intervals. c Concordance in aging changes in DNA methylation in the discovery and replication cohort. Venn diagram showing the significance of overlap between the discovery and replication cohorts in the genes with age-dependent changes in hemispheric asymmetry in DNA methylation. The $p$ value represents significance of overlap by hypergeometric test. $\mathbf{d}$ Top 10 genes involved in aging changes in hemispheric asymmetry. Genes affected by aging changes in DNA methylation were ranked using the discovery and replication DNA methylation datasets. Genes are listed by ranked order, and the heatmap shows adjusted rank significance score in each dataset 
changes in hemispheric asymmetry with aging $(q<0.05$, robust linear regression with contrasts; adjusted for sex, postmortem interval, and neuronal subtype proportion; $n=23$ PD-left, 34 PD-right, 25 control-left, 23 controlright; Fig. 4a; Additional file 5). When these sites were categorized according to diagnosis and hemisphere side, we observed that there are more aging changes in hemispheric asymmetry in the epigenomes of PD patients (5146 out of 5925 significant sites showing greater aging changes in PD than in controls; $p<10^{-15}$, Fisher's exact test; Fig. 4a). The left hemisphere of PD patients was particularly vulnerable to aging changes in DNA methylation, concordant with previous PD imaging studies [5, 52-55]. Interestingly, in both PD patients and controls, DNA methylation at enhancers and promoters became increasingly symmetrical across hemispheres with aging (Fig. 4b; Additional file 1: Figure S10). There was a reduction in asymmetry with aging at 4198 (70.9\%) cytosines in PD patients and 3818 (64.4\%) cytosines in controls, out of the 5925 age-associated sites $\left(p<0.001\right.$ and $p<10^{-7}$, respectively, Fisher's exact test; Fig. 4b). Though neuronal epigenomes across PD hemispheres became less asymmetric with age, left-right asymmetry in PD neuronal epigenomes persists even at advanced ages (Fig. 4b). Therefore, in aging, there is a progressive loss of hemispheric asymmetry in neuronal epigenomes in both controls and PD patients. Loss of epigenetic asymmetry between hemispheres of PD patients may contribute to bilateral symptomatic progression in PD [11].

We then identified the genes that had the most robust aging changes in hemispheric asymmetry in PD. We first determined the gene targets of the regulatory elements showing hemispheric differences in DNA methylation with aging and PD diagnosis (using the chromatin conformation analysis approach described above). There was strong overlap in the discovery and replication cohorts in the genes that showed aging changes in hemispheric asymmetry in PD relative to controls $\left(p<10^{-206}\right.$, hypergeometric test; Fig. 4c; Additional file 6). The genes with the most significant aging changes in hemispheric asymmetry in PD were then ranked based on consistency across the discovery and replication cohorts. Among the top genes, there were calcium/calmodulindependent protein kinase $2(C A M K 2 B)$, histone deacetylase 4 (HDAC4), and NCAM1, which have established roles in synaptic plasticity, neurotransmitter release, neurodevelopment, memory, and locomotor activity, as well as endothelin-converting enzyme-1 (ECE1), which degrades $\alpha$-synuclein pathology [56-60] (Fig. 4d). Hence, PD patients have aging changes in the hemispheric asymmetry of DNA methylation that affect genes involved in synaptic transmission, motor functions, and $\alpha-$ synuclein levels.

\section{Differential hemispheric asymmetry in the epigenome is associated with PD progression}

We examined the relationship between hemispheric asymmetry and disease course in PD patients. A short disease course was defined as less than 15 years of PD motor symptoms prior to death, while a long disease course exceeded 15 years. PD patients with either a short or a long disease course had similar ages at death (average age, short course: $77.4 \pm 1.7$ years; long course: $77.3 \pm 1.7$ years; both hemispheres of $n=14$ and 12 PD patients with short or long disease course, respectively; Additional file 2). In PD neurons, we examined the divergence of DNA methylation patterns between the symptom-dominant and non-dominant hemispheres with PD disease course. There were 2910 cytosine sites in enhancers and promoters showing changes in hemispheric asymmetry with PD duration $(q<0.05$, robust linear regression with contrasts, adjusting for age, sex, postmortem interval, brain hemisphere (left or right), and neuronal subtype proportion; Additional file 5). Hemispheric asymmetry in DNA methylation was greater in neurons of PD patients that had a long disease course $(p<0.001$, Student's $t$ test; Fig. $5 \mathrm{a})$. Hence, prominent epigenetic differences between hemispheres are linked to a slow PD progression. The greater hemispheric asymmetry in the epigenomes of PD patients with a longer disease course may explain the clinical observations that PD patients with highly lateralized symptoms have a slower disease progression than those with symmetrical symptoms [12].

Genes and pathways in neurons associated with differences in PD disease course were investigated. The gene targets of enhancers and promoters that showed hemispheric asymmetry changes in DNA methylation with PD disease course were identified (using chromatin conformation analysis of prefrontal cortex and the in silico regulatory element prediction tool). There were 3141 genes that had inter-hemispheric changes in epigenetic regulation with PD duration (Additional file 6). Pathway analysis determined that the length of disease course was related to epigenetic changes at genes affecting neurodevelopment, neuron differentiation, cell signaling, brain development, and potassium ion transport $(q<$ 0.05 , hypergeometric test; Fig. 5b). Hence, epigenetic mechanisms impacting brain development and neuronal communication may influence the progression of PD.

\section{PD risk genes exhibit hemispheric asymmetry in DNA methylation}

Finally, we sought to understand the contribution of PD risk genes to hemispheric asymmetry in PD. PD risk genes (determined by GWAS meta-analysis [50]) were identified among the genes that exhibited greater hemispheric asymmetry in PD, relative to controls, and that 
a

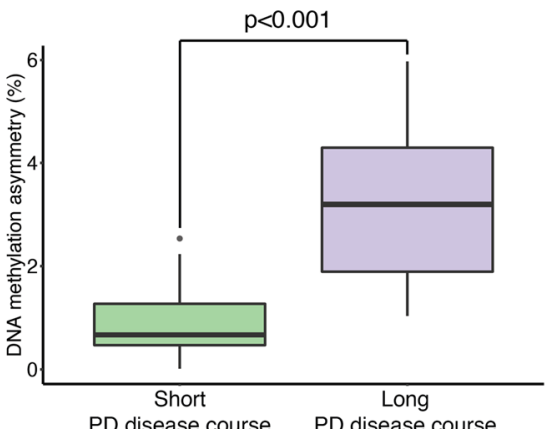

b

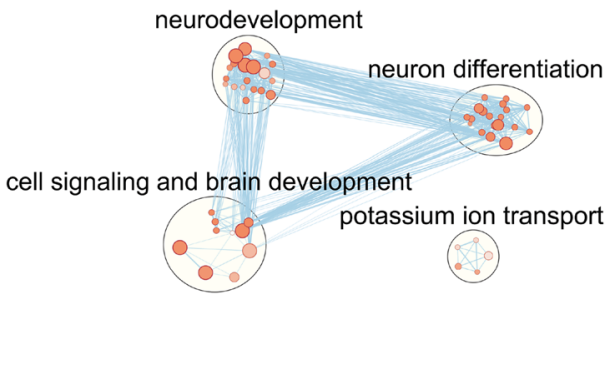

Fig. 5 Long PD disease course is associated with high levels of hemispheric asymmetry in DNA methylation. Significant DNA methylation changes associated with PD duration were identified ( $n=2910$ cytosine sites, $q<0.05$, robust linear regression with contrasts, controlling for age, sex, postmortem interval, neuronal subtype proportion, and brain hemisphere side). The extent of hemispheric asymmetry for cytosine sites associated with PD duration was determined in PD patients with a short ( $\leq 15$ years) or long (> 15 years) disease course $(n=14$ and 12 individuals, respectively). a The extent of hemispheric asymmetry in DNA methylation in PD patients with a short or long disease course. The boxplot center line is the median, the lower and upper limits are the first and third quartiles (25th and 75th percentiles), and the whiskers are $1.5 \times$ the interquartile range. $p<0.001$ is the difference between the short and long disease course groups in level of DNA methylation asymmetry, as determined by $t$-test. $\mathbf{b}$ Pathways differing between PD patients with a short or long disease course. Pathway enrichment analysis of genes with epigenetic differences associated with PD duration was performed by g:Profiler (nodes are $q<0.05$ pathways, hypergeometric test). Pathways were merged in Enrichment Map

were preferentially disrupted in the symptom-dominant PD hemisphere. We also examined the contribution of PD risk genes to aging-related and disease-durationrelated changes in hemispheric asymmetry in PD patients. Our DNA methylation, transcriptomic, and proteomic analyses were used to identify hemispheric differences in PD patients that involved PD risk genes (significant genes in analysis for Figs. 2, 3, 4, and 5; Additional file 6). We found 37 out of 72 PD risk genes showing more hemispheric asymmetry in PD relative to controls and/or showing greater differences in the PD hemisphere matched to symptom dominance (Fig. 6). These included genes involved in immune cell functioning and development (ITPKB, SATB1), axonal growth and synaptic signaling (ANK2, CAMK2D), and $\alpha-$ synuclein pathology (SNCA) [61-64]. Similar PD GWAS risk genes exhibited inter-hemispheric methylation changes with aging and were linked to a short duration of PD (Fig. 6). We also noticed that a region on chromosome 3 ( $300 \mathrm{~kb}$, spanning from ALAS1 to STAB1) was consistently involved in hemispheric asymmetry in PD across datasets, suggesting a combined epigenetic and genetic disruption of this area in PD. These findings suggest that epigenetic changes at PD risk genes contribute to lateralized hemisphere dysregulation in PD.

We also determined the effects of cis-acting genetic variation on the DNA methylation changes involving hemispheric asymmetry in PD. SNPs that were proximal to the cytosine sites profiled in our bisulfite sequencing data were identified; $69 \%$ of cytosines related to hemispheric asymmetry in $\mathrm{PD}$, and $70 \%$ of all cytosines profiled in our study, had one or more SNPs identified within $\pm 500 \mathrm{~kb}$. We then performed a methylation quantitative trait loci (me-QTL) analysis to examine the effects of genotype on DNA methylation and determined that 11,507 out of 564,294 cytosines had a meQTL association $(q<0.05$, robust linear regression, after adjusting for diagnosis, hemisphere, age, sex, postmortem interval, and neuronal subtypes). We found that cytosine sites relevant to hemispheric asymmetry in PD had a significant increase in SNP associations relative to all cytosine sites profiled $(5.3 \%$ of cytosines related to hemispheric asymmetry in PD vs. $2.0 \%$ of background cytosines, $p<$ $10^{-15}, \chi^{2}$ test). In total, we found genetic variation to be associated with 211 genes with inter-hemispheric differences in DNA methylation in PD (Additional file 11). Interestingly, pathway analysis revealed that these genes affect neurodevelopment and NCAM1-mediated neurite outgrowth, synapse assembly, and neuronal survival (Additional file 1: Figure S11; Additional file 11). Thus, genetic factors may drive a number of DNA methylation changes involved in hemispheric asymmetry in PD.

\section{Discussion}

Although hemispheric asymmetry is a fundamental biological feature of the human brain, its determinants remain unclear. Here, we demonstrate that the epigenetic states of neurons differ between hemispheres and may play a role in the lateralization of brain functions. The prominent inter-hemispheric differences in the epigenome, transcriptome, and proteome identified in this study signify that hemisphere side should be considered in future molecular studies of brain health and disease. 


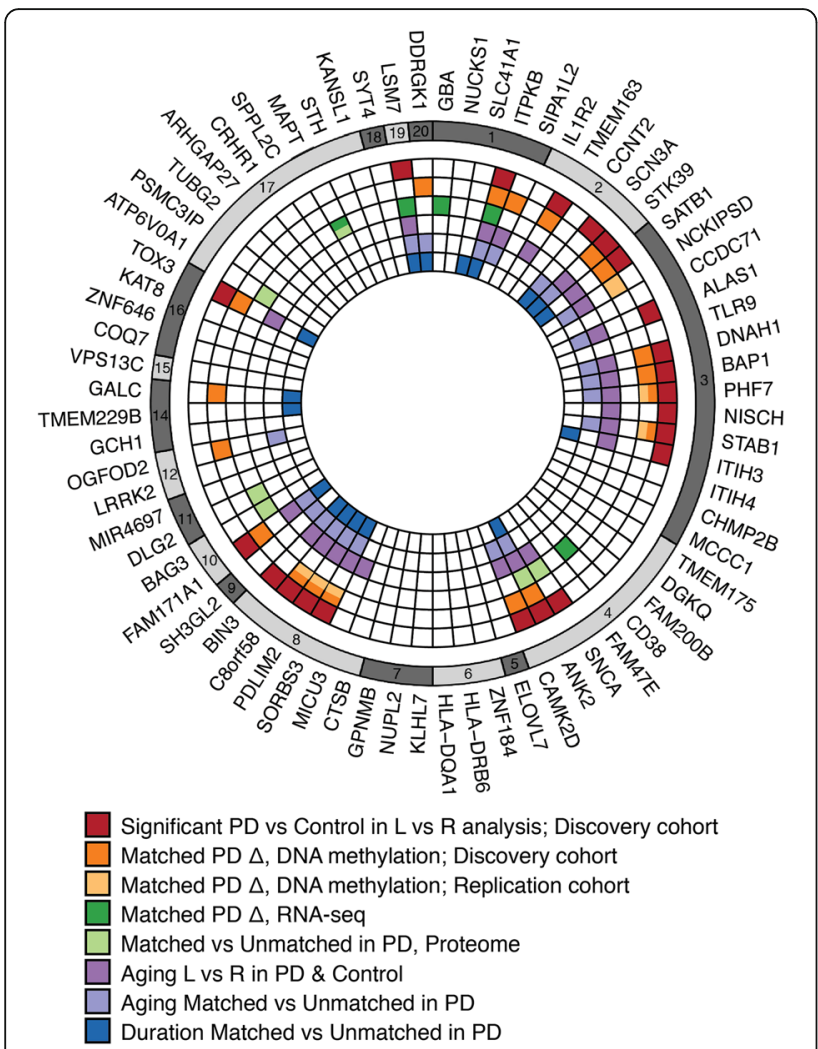

Fig. 6 PD risk genes contribute to hemispheric asymmetry in neurons of PD patients. PD risk genes identified by GWAS [50] were found to exhibit differential hemispheric asymmetry in PD patients relative to controls, were preferentially dysregulated in the PD symptom-dominant hemisphere, and were involved in hemispheric asymmetry changes occurring with aging and PD disease course. Genes identified in eight independent analyses that include epigenetic, transcriptomic, or proteomic data are presented. The plot summarizes PD risk genes with (1) left-right hemispheric differences in PD relative to controls that involve epigenetic changes at cisregulatory elements (red); ( 2 and 3 ) epigenetic dysregulation that is greater in the symptom-dominant hemisphere than in the nondominant hemisphere (relative to control hemispheres), as determined in discovery cohort (orange) and replication cohort (yellow); (4 and 5) disruption in PD that is associated with symptom lateralization, as determined by analysis of the transcriptome (dark green) and proteome (light green); (6) aging changes in left-right hemispheric asymmetry in epigenetic regulation in PD and controls (purple); (7) aging changes in epigenetic regulation between the symptom-dominant and non-dominant hemisphere in PD (indigo); (8) hemisphere asymmetry in DNA methylation associated with length of PD disease course (blue). Colored boxes represent significant changes affecting the PD risk gene

Our analysis showed hemispheric differences in DNA methylation in healthy cortical neurons that were mainly driven by differential accumulation of $\mathrm{CpH}$ methylation. $\mathrm{CpH}$ methylation at enhancers and promoters was greater in neurons of the left hemisphere. At enhancers, $\mathrm{CpH}$ methylation is correlated with reduced gene transcript levels [21, 23]. Neurons exhibit a prominent gain in $\mathrm{CpH}$ methylation in the first 5 years of life, during a period of major synaptic restructuring [20, 65, 66]. Changes in $\mathrm{CpH}$ methylation status in early life have been implicated in synapse formation, neurotransmission, and alternative splicing $[20,65]$. Neuronal $\mathrm{CpH}$ methylation changes with aging have also been associated with neurodegenerative and neurocognitive disorders [32, 65]. In our study of adult brain neurons, hemispheric asymmetry in $\mathrm{CpH}$ methylation preferentially affected genes involved in neurodevelopment, synaptic organization, and brain diseases, including neurodegenerative illnesses. Neuronal asymmetry in $\mathrm{CpH}$ methylation at gene regulatory elements may instill a differential vulnerability of hemispheres to synaptic dysfunction and neuronal loss that lead to neurodegenerative diseases. In support, hemispheric asymmetry in DNA methylation for PD patients was largely driven by changes in $\mathrm{CpH}$ methylation.

Our study shows that hemispheric asymmetry of the epigenome is exaggerated in PD, with greater methylation abnormalities observed in the hemisphere matched to the symptom-dominant side. A major challenge in epigenetic studies is differentiating epigenetic changes that are causal to disease from those that arise as a consequence of a non-shared environment. Because we examined neurons in each brain hemisphere of the same individual, we were able to delineate epigenetic differences that preferentially appear on the symptom-dominant side of the PD brain from those appearing in both hemispheres. As such, we identified epigenetic changes, genes, and pathways most relevant to the presentation of PD clinical symptoms. We reinforced our findings with transcriptomic and proteomic analyses and replicated our results in an independent cohort. Finally, hemispheric asymmetry in the epigenome impacts PD risk genes identified by GWAS [50], further supporting that epigenetic dysregulation at these genes could contribute to disease pathobiology.

The factors rendering neurons more vulnerable in one brain hemisphere over the other is one of the most enigmatic puzzles of PD. Previously, it has been proposed that symptom lateralization in PD is related to body side preference (handedness), though this effect is relatively modest and not replicated in all studies [2, 67-69]. Our epigenetic, transcriptomic, and proteomic analyses of hemispheric differences in PD, relative to controls, converges on gene pathways involved in neurodevelopment, immune activation, and synaptic transmission. Analysis of phenotypic pathways associated with hemispheric asymmetry in PD were strongly related motor dysfunction, further supporting the relevance of the genes identified in this study to PD. Specifically, we found that hemispheric asymmetry in PD was linked to the dysregulation of NCAM1. NCAM1 exerts a regulatory role on the development and survival of dopaminergic neurons [70] and also impacts immune responses [71]. Our 
analysis of genetic-epigenetic interactions indicates that NCAM1 abnormalities in PD may have a genetic basis and consequently may precede disease onset. In addition, we found hemispheric differences in $\alpha$ synuclein, which has a central role in Lewy pathology in PD [41]. In experimental models, selective vulnerability of brain regions to the spread of synucleinopathy and neuronal death was closely correlated with neuronal connectivity and endogenous $\alpha$-synuclein levels [72, 73]. Hence, molecular asymmetries, particularly those affecting neuronal development and survival, neurosignaling, and immune activation, may render one hemisphere more vulnerable to degenerative processes in PD.

In advanced age, there is a convergence in neuronal epigenomes between hemispheres. This could explain the reduction in hemispheric asymmetry observed in functional neuroimaging studies of old as compared to young adults $[74,75]$. We also found that the left hemisphere of the PD brain had greater epigenetic dysregulation than the right hemisphere, which is consistent with PD imaging studies $[74,75]$. The convergence of hemisphere epigenomes in aging PD patients may explain why symptoms become more bilateral as PD progresses [11]. We also found that a long PD duration was associated with greater hemispheric asymmetry in DNA methylation. Clinically, PD patients with symmetrical symptom onset are prone to rapid disease progression [12], and in our study, rapid disease progression was associated with less asymmetry in DNA methylation that affects genes involved in neurodevelopment and cell signaling.

\section{Conclusions}

Based on the results of our multi-omics study, we postulate that hemispheric asymmetry in PD results from the differential regulation of genes involved in nervous system development, immune signaling, and synaptic transmission (Fig. 7). Lateralization is developed early in the brain, and neuronal progenitors can shape the recruitment and positioning of brain-resident immune cells, the microglia [76, 77]. These immune cells are sessile, show regional differences in the brain $[78,79]$, and have dynamic processes that shape synaptic structure, maturation, and signaling $[80,81]$. In addition, hemispheric differences in select genes influencing neuronal survival may contribute to asymmetric responses to PD pathogenic processes. Hence, it is possible that earlyestablished differences in neuronal structure and survival, in combination with lifelong differences in immune activity and neurotransmission across hemispheres, lead to unilateral vulnerability, which may explain the corresponding unilateral presentation of PD symptomatology.

\section{Methods}

No statistical methods were used to predetermine sample size.

\section{Human tissue samples}

Human prefrontal cortex tissue for this study was obtained from the Parkinson's UK Brain Bank, NIH NeuroBioBank, and Michigan Brain Bank, with approval from the ethics committee of the Van Andel Research Institute (IRB \#15025). For each individual, we had information on demographics (age, sex, ethnicity), brain hemisphere, tissue quality (post-mortem interval), side of symptom predominance, PD duration, and pathological staging (Additional file 2). Control individuals had pathologically normal brains (and verified to have no brain Lewy body pathology). PD cases were pathologically confirmed to have brain Lewy body pathology. We examined two independent cohorts of samples; the discovery and replication cohort. The discovery cohort included 105 individuals: 48 controls (brain hemisphere left, 25; right, 23) and 57 PD patients (brain hemisphere left: 23; right: 34$)$. For the PD patients in the discovery cohort, 37 had information about the side of symptom predominance (hemisphere matched to side of symptom dominance, 17; unmatched, 20). The replication cohort included both hemispheres of 57 individuals: 31 controls and 26 PD patients, and prefrontal cortex tissue was obtained from the same area of both hemispheres for each individual. The side of symptom predominance was known for all the PD patients in the replication cohort. Information on handedness was not available for controls and PD patients. Neurons of the prefrontal cortex were selected for this study because (1) this brain region plays an important role in PD [82], (2) pathology does spread to this brain region [83], and (3) neurons remain present in the PD prefrontal cortex [84] (in contrast to the substantia nigra, which has undergone severe neurodegeneration [11]).

\section{Isolation of neuronal nuclei by flow cytometry}

Neuronal nuclei were isolated from the human prefrontal cortex using flow cytometry [32, 46]. Freshfrozen prefrontal cortex tissue (200-300 mg) was rinsed and finely chopped in $2 \mathrm{~mL}$ of PBSTA Buffer $(0.3 \mathrm{M}$ sucrose, $1 \times$ Dulbecco's PBS (Gibco), $3 \mathrm{mM} \mathrm{MgCl}_{2}$ ). The sample was then homogenized on ice for three intervals of $5 \mathrm{~s}$ (BioSpec Tissue Tearor, on lowest setting). For each sample, $40 \mu \mathrm{L}$ of $10 \%$ Triton $\mathrm{X}-100$ was added to the homogenate and incubated for $15 \mathrm{~min}$. Next, the tissue homogenate was transferred to a dounce homogenizer (Kimble) and homogenized eight times. The homogenate was filtered through Miracloth (Calbiochem) and passed through a sucrose cushion (1.4 M, $1 \times$ Dulbecco's PBS (Gibco), 0.1\% TritonX-100, $3 \mathrm{mM}$ 


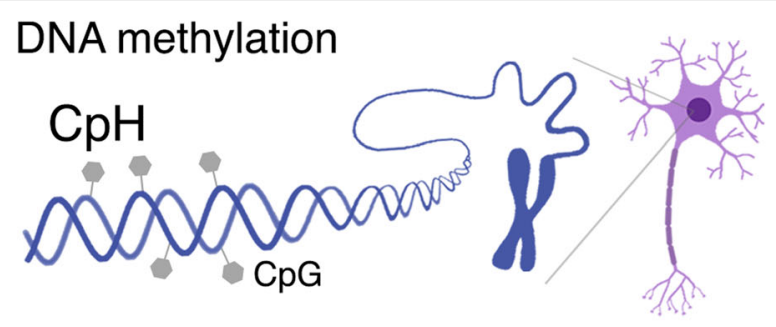

\section{Healthy}

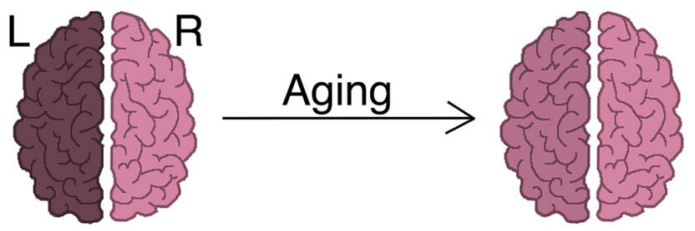

PD
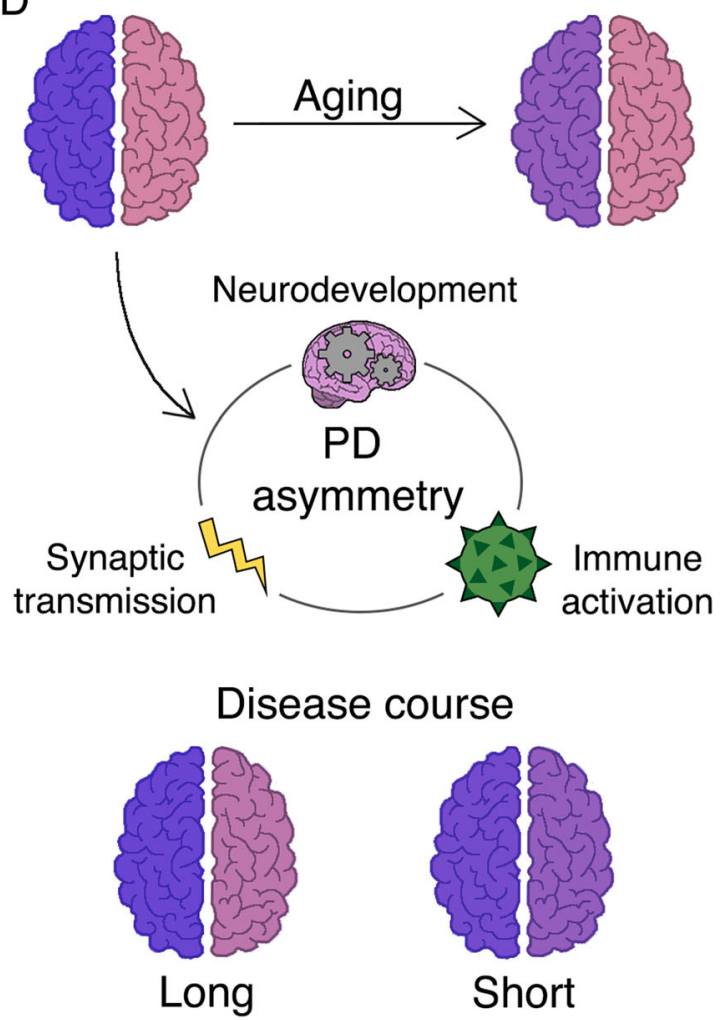

Fig. 7 Schema of hemispheric asymmetry in the epigenome of the healthy and PD brain. Neurons of the human prefrontal cortex exhibit prominent hemispheric asymmetry in DNA methylation. DNA methylation levels are higher in neurons of the left hemisphere, a difference driven largely by the accumulation of $\mathrm{CpH}$ methylation. Compared to neurons in the control brain, neurons in the PD brain possess considerably greater hemispheric asymmetry, again primarily driven by differential $\mathrm{CpH}$ methylation. Hemispheric asymmetry in PD involves DNA methylation abnormalities that are more prominent on the hemisphere matched to the side of symptom dominance. Aberrant hemispheric asymmetry and symptom lateralization in PD is related to disruption of genes affecting neurodevelopment, immune activation, and synaptic transmission. In aging, neuronal epigenomes exhibit a decrease in hemispheric asymmetry. The convergence of neuronal epigenomes in PD with aging may contribute to the bilateralization of PD symptoms over time, though hemispheric asymmetry in DNA methylation persists even at advanced ages. Epigenetic asymmetry between hemispheres is also linked to disease progression: PD patients with long (>15 years) disease courses have greater asymmetry than patients with short ( $\leq 15$ years) disease courses. Shading of brain hemispheres represents asymmetry in DNA methylation between paired hemispheres (shading represents DNA methylation status) 
$\mathrm{MgCl}_{2}$ ) by centrifugation at $3000 \times g$ for $30 \mathrm{~min}$ at $4{ }^{\circ} \mathrm{C}$. After removing the supernatant, the pelleted nuclei were incubated for $15 \mathrm{~min}$ in $800 \mu \mathrm{L}$ of blocking buffer: $1 \times$ PBS, $1.25 \%$ goat serum (Gibco), $3 \mathrm{mM} \mathrm{MgCl}_{2}$, and $0.0625 \%$ BSA (Thermo Fisher Scientific). The nuclei were then gently resuspended and mixed with antiNeuN antibody (1:500, Abcam) and incubated for at least $30 \mathrm{~min}$ on ice. Immediately before sorting, $10 \mu \mathrm{L}$ of 7-AAD or DAPI (Thermo Fisher Scientific, SigmaAldrich) was added to each sample, and nuclei samples were filtered through a $41-\mu \mathrm{m}$ filter (Elko Filtering Co.). Samples were sorted on a MoFlo Astrios in the Flow $\mathrm{Cy}-$ tometry Core of the Van Andel Research Institute, using the gating strategy described in Additional file 1: Figure S1. After sorting, nuclei were pelleted in $10 \mathrm{~mL}$ Dulbecco's $1 \times$ PBS (Gibco), $0.3 \mathrm{M}$ sucrose, $5 \mathrm{mM} \mathrm{CaCl}_{2}$, and 3 $\mathrm{mM} \mathrm{MgCl}$. Samples were mixed by inverting and incubated on ice for $15 \mathrm{~min}$ before centrifuging at $2500 \times g$ for $10 \mathrm{~min}$. The supernatant was removed, and pellets were frozen at $-80^{\circ} \mathrm{C}$ until DNA isolation. Neuronal nuclei DNA was isolated using standard phenol-chloroform methods.

\section{Fine-mapping of DNA methylation with bisulfite padlock probe sequencing}

The bisulfite padlock probe sequencing technique was used for the targeted quantification of DNA methylation with single-nucleotide resolution at enhancers and promoters in neurons of the human prefrontal cortex [32, 85]. Human brain enhancers and promoters were identified using the EpiCompare tool [86], which identifies tissue/cell type gene regulatory elements based on chromatin state data defined by the ChromHMM tool [87] from the RoadMap Epigenomics Project [88]. Enhancers and promoters were defined based on the 18state ChromHMM model [87]. Our study included all genic, active, weak, or poised/bivalent enhancers (7_ EnhG1, 8_EnhG2, 9_EnhA1, 10_EnhA2, 11_EnhWk, 15_ EnhBiv). We also included all promoters that were active, near a transcription start site, or poised/bivalent (1 TssA, 2_TssFlnk, 3_TssFlnkU, 4_TssFlnkD, 14_TssBiv; for E073, E072, and E074). Enhancers and promoters significantly enriched in the adult brain are from the Tissue Specific Enhancers website (https://epigenome. wustl.edu/TSE/browse.php). We also included all enhancers and promoters present in adult prefrontal cortex (E073), inferior temporal lobe (E072), and substantia nigra (E074).

Padlock probes $(n=59,009)$ for bisulfite analysis targeted the unique (non-repetitive) enhancer and promoter regions on both forward and reverse DNA strands. Padlock probes were designed using ppDesigner (v2.0) [89] with the human GRCh37/hg19 genome. Probe sequences are described in Additional file 3.
Padlock probes were synthesized using a programmable microfluidic microarray platform (CustomArray, Inc.) and were prepared and purified for experiments, as described [85].

DNA methylation fine-mapping using the bisulfite padlock probe sequencing approach was performed as previously described $[32,85]$. In brief, genomic DNA for each sample was bisulfite-converted and purified using the EZ DNA Methylation Kit (Zymo Research). The bisulfite-converted DNA (200 ng) was hybridized to the padlock probes $(1.5 \mathrm{ng})$. Targeted regions were extended using PfuTurbo $\mathrm{Cx}$ (Agilent Technologies), and circularization was completed using Ampligase (Epicenter). Non-circularized DNA was digested using an exonuclease cocktail, and the remaining target circularized DNA was amplified using a common linker sequence in the padlock probe. Libraries were PCR-amplified, purified with AMPure XP beads (Beckman Coulter A63881), pooled in equimolar amounts, and further purified using a QIAquick Gel Extraction kit (Qiagen). Libraries were quantified using the Qubit dsDNA HS Assay Kit (Thermo Scientific) and qPCR (Kapa Biosystems) on a ViiA 7 Real-time PCR system (Applied Biosystems). Next-generation sequencing of the libraries was performed by the Epigenetics Lab at the Centre for Addiction and Mental Health in Toronto, Canada, on an Illumina HiSeq 2500 machine in HiOutput mode. Library sequencing was done across 3 flow cells (24 lanes) for the discovery cohort and across 2 flow cells (16 lanes) for the replication cohort, yielding 25-40 million reads/sample.

\section{Epigenomic data analysis}

We examined DNA methylation status at every cytosine site $(\mathrm{CpG}$ and $\mathrm{CpH})$ covered by padlock probes targeting 35,288 regulatory regions across the genome with a custom pipeline $[32,85]$. This pipeline was used for both the discovery $(n=108$ unique samples, 17 technical replicates) and replication ( $n=114$ unique samples, 12 technical replicates) cohorts. First, we removed low-quality bases and performed adapter trimming of bisulfitetreated sequencing reads using Trimmomatic- 0.32 for the discovery cohort or Trim Galore (v0.4.4) for the replication cohort. Bismark (v0.17.0) [90] was used to align reads to the target reference genome (GRCh37/hg19) and perform methylation calls. Methylation calls were included only for cytosines with a minimum read depth of 30x. Bisulfite conversion efficiency was $99.14 \pm$ $0.005 \%$ in the discovery cohort and $99.28 \pm 0.007 \%$ in the replication cohort (averaged CC methylation per sample). We excluded 5 samples in the discovery cohort (3 unique samples and 2 replicates) and 3 samples in the replication cohort (3 unique samples) from further analyses due to poor inter-sample correlations (>10\% 
difference). Technical replicates confirmed a high reproducibility in the sample-level DNA methylation correlation analysis (average $\mathrm{R}$ for the discovery cohort $0.94 \pm$ 0.007; average $\mathrm{R}$ for the replication cohort: $0.97 \pm 0.003$; Additional file 1: Figure S2). DNA methylation calls at each site were merged for matched technical replicate samples. Cytosine sites with missing (unknown) DNA methylation calls in more than $30 \%$ of samples were excluded. We also removed cytosine sites overlapping common SNPs (minor allele frequency $\geq 0.05$ ), as identified by the 1000 Genomes Project (phase 3 v5a 20130502 release for chr1 chr22, v1b 20130502 for chrX; all populations and European populations) [91]. $\mathrm{CpG}$ and $\mathrm{CpH}$ sites that had stable DNA methylation calls (DNA methylation status of $0 \%$ or $100 \%$ or NA) in more than $50 \%$ of samples were excluded from further analysis. At the end of these preprocessing steps, the discovery cohort had 105 samples with 633,803 CpGs/ $\mathrm{CpHs}$ and the replication cohort had 111 samples with $815,367 \mathrm{CpGs} / \mathrm{CpHs}$ that consisted of quality-controlled, genome-wide methylation data retained for downstream analysis (Additional file 1: Figure S2; Additional file 2).

\section{Neuronal subtype proportion}

In the prefrontal cortex, $70-85 \%$ of neurons are excitatory glutamatergic neurons, while the remaining 15-30\% are inhibitory GABAergic neurons [92]. Our DNA methylation analysis adjusts for variation in neuronal subtypes. We performed cell-type deconvolution using CIBERSORT [93] and reference neuronal subtypespecific markers (gene body $\mathrm{CpH}$ methylation) provided in a single-cell DNA methylome analysis of the human frontal cortex [92]. For the reported 1012 neuronal subtype gene signatures, we examined averaged $\mathrm{CpH}$ methylation within gene bodies $( \pm 100 \mathrm{~kb})$ and found 563 neuronal subtype gene signatures for the discovery cohort and 610 signatures for the replication cohort. Using the neuronal subtype signature matrix (gene $\mathrm{CpH}$ markers), CIBERSORT was run with 100 permutations. We did not find any significant differences in any type of neuron (Additional file 4). To control for neuronal subtype variation in our DNA methylation analysis, we used the proportion of glutamatergic relative to GABAergic subtypes for each sample.

\section{Statistical analysis for differentially methylated sites}

DNA methylation analysis for the discovery and replication cohort involved multivariate robust linear regression models with empirical Bayes from the limma (v3.30.13) statistical package [94]. DNA methylation was transformed from $B$ values to $M$ values using lumi (v2.30.0) [95]. Because the replication cohort data has both hemispheres from the same individual, we added in the limma model a blocking factor to define samples from the same individual and a correlation coefficient determined by the duplicateCorrelation function. For each dataset and contrast, $p$ values were adjusted with a Benjamini-Hochberg correction for multiple testing and those with FDR $q<0.05$ were deemed significant.

\section{Analysis of hemispheric asymmetry in DNA methylation In controls, PD, and aging (model 1)}

To analyze hemispheric asymmetry in DNA methylation, the linear model matrix for $\mathrm{CpG} / \mathrm{CpH}$ methylation $(M)$ as a dependent variable is:

$$
\begin{aligned}
M \sim & \text { brain hemisphere }(\text { left or right }) \\
& \times \text { diagnosis }(\text { control or PD }) \times \text { age }+ \text { sex } \\
& + \text { postmortem interval } \\
& + \text { neuronal subtype proportion }
\end{aligned}
$$

We used the contrasts.fit function to identify sites exhibiting hemispheric asymmetry in controls with the following contrasts matrix: right brain hemisphere in controls - left brain hemisphere in controls. To identify sites exhibiting hemispheric asymmetry in PD patients relative to controls we used the contrasts matrix: (right brain hemisphere in PD - left brain hemisphere in PD) - (right brain hemisphere in controls - left brain hemisphere in controls). To determine whether hemispheric asymmetry is greater in PD patients or controls, the difference between the absolute fold change of hemispheric asymmetry in PD patients and controls was determined.

To identify $\mathrm{CpG} / \mathrm{CpH}$ sites exhibiting age-dependent DNA methylation differences in PD patients and controls, we used the contrast matrix: [(right brain hemisphere in PD at age max - left brain hemisphere in PD at age max) - (right brain hemisphere in PD at age min - left brain hemisphere in PD at age min)] - [(right brain hemisphere in controls at age max-left brain hemisphere in controls at age max) - (right brain hemisphere in control at age min - left brain hemisphere in control at age min)]. Age max and min refer to the highest and lowest age value in the cohort, respectively. To determine whether aging changes in hemispheric asymmetry are greater in PD or controls, the absolute fold change of aging differences in hemispheric asymmetry in PD was compared with that of controls.

\section{Related to side of symptom dominance (model 2)}

DNA methylation changes were examined in PD hemispheres matched or unmatched to side of PD symptom predominance in comparison to control hemispheres. The linear model matrix for $\mathrm{CpG} / \mathrm{CpH}$ methylation as a dependent variable is: 


$$
\begin{aligned}
M \sim & \text { hemisphere category (matched, unmatched, or control) } \\
& + \text { brain hemisphere (left or right })+ \text { age }+ \text { sex } \\
& + \text { postmortem interval + neuronal subtype proportion }
\end{aligned}
$$

We used the contrasts.fit function to identify cytosine sites exhibiting DNA methylation changes in the matched or unmatched hemisphere of PD patients relative to both hemispheres of controls using the contrasts matrix: Matched PD hemisphere - control hemisphere, and separately, unmatched PD hemisphere-control hemisphere. We identified cytosine sites that were significantly altered in the matched PD hemisphere relative to controls and that exhibited greater DNA methylation fold changes than the unmatched hemisphere.

\section{Aging of the symptom-dominant and non-dominant PD hemisphere (model 3)}

Aging changes in DNA methylation in the symptomdominant and non-dominant hemisphere of PD patients were determined. The linear model matrix for $\mathrm{CpG} /$ $\mathrm{CpH}$ methylation as a dependent variable is:

$$
\begin{aligned}
M \sim & \text { hemisphere category } \times \text { age }+ \text { brain hemisphere } \\
& + \text { sex }+ \text { postmortem interval } \\
& + \text { neuronal subtype proportion }
\end{aligned}
$$

We determined aging changes in hemispheres matched or unmatched to side of symptom dominance using the contrasts matrix: (matched PD hemisphere at age max-matched PD hemisphere at age min) - (unmatched PD hemisphere at age max-unmatched PD hemisphere at age min).

\section{In response to PD disease course (model 4)}

Analysis of changes in hemispheric asymmetry of DNA methylation in response to PD disease course was performed using the data from PD patients in the replication cohort, consisting of both hemispheres from the same PD patients with a known side of symptom predominance. The linear model matrix for $\mathrm{CpG} / \mathrm{CpH}$ methylation as a dependent variable is:

$$
\begin{aligned}
M \sim & \text { hemisphere category } \times \text { disease course } \\
& + \text { brain hemisphere }+ \text { age }+ \text { sex } \\
& + \text { postmortem interval } \\
& + \text { neuronal subtype proportion }
\end{aligned}
$$

We identified cytosine sites exhibiting changes in hemisphere asymmetry with disease course using the contrasts matrix: (matched PD hemisphere at duration max - matched PD hemisphere at duration min) - (unmatched PD hemisphere at duration max - unmatched PD hemisphere at duration min). Duration max and min refer to the longest and shortest duration value in the cohort, respectively.

\section{Gene annotation and enrichment analysis}

Because enhancer elements dynamically regulate gene expression through three-dimensional physical interactions, we analyzed chromatin interaction data to reveal the gene targets of enhancers relevant to hemisphere asymmetry. For this analysis, we used promoter-centric chromatin interactions identified in human cortical neurons (93,290 interactions) [47] and in human excitatory neurons (73,890 interactions) [48]. Gene annotation involved identifying interactions with gene promoters (TSS $\pm 2 \mathrm{~kb}$ ). To further identify proximal interactions with gene targets, we used the GREAT (v4.0.4) software [49]. Gene annotation was performed for the gene targets of the significant cytosine sites in our analysis and for the background, consisting of gene targets for all cytosines included in our analysis. The background in the discovery dataset was 9005 genes and in the replication dataset was 9528 genes.

In the Manhattan plot comparing hemispheric asymmetry in PD patients relative to controls (Fig. 2a), PD risk genes identified in familial studies and/or by GWAS [50] are denoted. For figure clarity, we show the PD risk genes that have hemispheric differences in DNA methylation at least three cytosines in enhancers/promoters. Direction of DNA methylation asymmetry determined by the direction of the majority of significant cytosines, with ties resolved by the most significant cytosine.

\section{RNA-sequencing}

We used RNA-seq to profile the mRNA transcriptome in the prefrontal cortex of PD hemispheres with known side of symptom predominance $(n=36$ individuals: 12 matched PD hemisphere, 11 unmatched PD hemisphere, and 13 controls). Fresh-frozen human prefrontal cortex tissue $(25-50 \mathrm{mg}$ ) was lysed and homogenized in $1 \mathrm{~mL}$ of TRIzol (Invitrogen) in a Precellys Lysing Kit CKMix tubes using a MiniLys homogenizer (Bertin Instruments, two intervals of $10 \mathrm{~s}$ on the highest setting with $15 \mathrm{~s}$ in between). Total RNA was isolated using the standard TRIzol protocol and re-suspended in $85 \mu \mathrm{L}$ of ultrapure distilled water (Invitrogen). DNase treatment was performed with the RNase-Free DNase kit (Qiagen) using $5 \mu \mathrm{L}$ DNase I and $10 \mu \mathrm{L}$ RDD buffer, followed by a column cleanup using the RNeasy Mini kit (Qiagen) with two additional washes (75\% ethanol) before elution. RNA quantity was assessed by Nanodrop 8000 (Thermo Scientific) and quality was assessed with an Agilent RNA 6000 Nano Kit on a 2100 Bioanalyzer (Agilent Technologies, Inc.). Libraries were prepared by the Van Andel Genomics Core from $500 \mathrm{ng}$ of total RNA using the KAPA RNA HyperPrep Kit with RiboseErase (v1.16) (Kapa Biosystems). RNA was sheared to an average of $300 \mathrm{bp}$. Prior to PCR amplification, cDNA fragments 
were ligated to NEXTflex Adapters (Bioo Scientific). The quality and quantity of the finished libraries were assessed using a combination of Agilent DNA High Sensitivity chip (Agilent Technologies Inc.) and QuantiFluor dsDNA System (Promega Corp.). Individually, indexed libraries were pooled, and 75-bp single-end sequencing was performed on an Illumina NextSeq 500 sequencer, with all libraries run across four flow cells to return a minimum read depth of 40 million read pairs per library. Base calling was done by Illumina NextSeq Control Software (NCS; v2.0), and the output of NCS was demultiplexed and converted to FastQ format with Illumina Bcl2fastq (v1.9.0).

Trim Galore (v0.11.5) was used to trim the 75-bp single-end reads prior to genome alignment. STAR (v2.3.5a) [96] index was generated using Ensemble GRCh37.p13 primary assembly genome and the Gencode v19 primary assembly annotation. Read alignment and gene counts were performed using STAR [96]. The gene count matrix was imported into R (v3.5.1) and low expressed genes (counts per million $<1$ in all samples) were removed prior to trimmed mean of $M$ values normalization in edgeR (v3.16.5) [97]. There was one sample excluded from further analyses due to poor inter-sample correlations ( $>10 \%$ difference). At the end of these pre-processing steps, 36 samples with 14,121 genes were retained for downstream analysis. The limma (v3.30.13) statistical package [94] was used to transform the count matrix to log2-counts per million. A generalized linear model was then used to determine gene transcript level changes in PD hemispheres that are relevant to symptom lateralization (hemisphere category: matched PD, unmatched PD, or control), adjusting for brain hemisphere, age, sex, RIN, and neuron proportion. In addition, we controlled for other sources of variation using RUVSeq (v1.18.0) [51]. We used contrasts to identify differentially expressed genes between matched PD hemisphere vs. control as well as unmatched PD hemisphere vs. control. $p$ values were adjusted for multiple testing correction using the Benjamini-Hochberg method.

Our RNA-seq analysis is corrected for the proportion of neuronal cells in each sample. Cell-type deconvolution was performed using CIBERSORT [93], which performs a linear support vector machine learning algorithm on normalized cell type-specific count data. In this approach, we used a gene signature matrix (involving 903 cell-specific marker genes) derived from single-cell RNA-seq measures in adult human brain cells (signature matrix [98]; source [99]). CIBERSORT was run with 100 permutations, and values were used for the neuronal cell composition adjustment in the generalized linear model for transcriptomic analysis.

\section{DNA methylation status correlated with target gene mRNA levels}

We determined whether enhancers and promoters exhibiting DNA methylation changes related to PD symptom lateralization had corresponding changes in target gene transcript levels ( $n=36$ individuals). For this analysis, we used DNA methylation and RNA-seq data for the same individuals and examined genes exhibiting significant and non-significant DNA methylation changes at their enhancer/promoter in the PD hemisphere matched to symptom dominance. The most significant differentially methylated sites for each target gene were used. For each gene, the diagnosis effect in the RNA-seq data was determined as the residual gene expression in a linear model adjusting for brain hemisphere, age, sex, RIN, neuron subtype proportion, and other sources of variation. For each gene, the diagnosis effect in the DNA methylation was determined as the residual in the linear model adjusting for brain hemisphere, age, sex, postmortem interval, and neuronal subtype proportion. The diagnosis effect in the RNA-seq data was then correlated to that of the DNA methylation data, examining genes with significant $(n=111$ at $q<0.05)$ and non-significant $(n=6038)$ DNA methylation changes, separately. Significant association between changes in DNA methylation at gene regulatory elements and changes in corresponding transcript levels in PD as compared to nonsignificant genes was determined by the interaction term in the linear regression.

\section{Pathway enrichment analysis}

Pathway enrichment analysis for the genes involved in DNA methylation asymmetry in the control brain was done with MetaCore (https://clarivate.com/products/ metacore/) and was relative to background genes. Pathway analysis integrating epigenetic and transcriptomic data was performed to identify gene pathways involved in hemispheric asymmetry and symptom lateralization in PD. Pathway analysis was done for DNA methylation data using g:Profiler [100] and for transcriptomic data using GSEA pre-ranked (v3.0) [101] with Human GOBP_AllPathways_no_GO_iea_November_01_2017_ symbol.gmt from [http://baderlab.org/GeneSets]. Analysis of DNA methylation data identifying pathways involved in hemispheric asymmetry changes with $\mathrm{PD}$ disease course was done by g:Profiler [100]. Pathway networks were determined by EnrichmentMap [102] and annotated by AutoAnnotate [103] in Cytoscape (v3.7.1). The protein-protein interaction network was performed using STRING (v11.0) [104]. Finally, pathway enrichment analysis for the genes with genetic variation associated with inter-hemispheric differences in DNA methylation in PD was done using MetaCore (https:// clarivate.com/products/metacore/). 


\section{Gene ranking}

We identified genes having DNA methylation changes most strongly associated with PD symptom lateralization. For this analysis, we used the epigenetic and transcriptomic data from our discovery cohort along with the epigenetic data from our replication cohort. We identified in each dataset the genes preferentially altered in the PD symptom-dominant hemisphere. For DNA methylation data, genes were ranked by $p$ value of the most significant site. For RNA-seq data, genes in PD were ranked by $p$ value. We then determined the genes consistently exhibiting greater DNA methylation in symptom-dominant hemisphere of PD patients across DNA methylation and RNA-seq datasets using the aggregateRanks function from the RobustRankAggreg package (v1.1) [105]. We also identified the genes with the most robust aging changes in hemispheric asymmetry in PD patients. As above, genes with significant aging changes in DNA methylation across hemispheres and with differential aging in PD were ranked based on consistency across discovery and replication cohorts, with the most robustly altered genes across datasets determined using the RobustRankAggreg package [105].

\section{Mass spectrometry and proteomics analysis}

Quantitative proteomic analysis of the prefrontal cortex of PD patients and controls ( $n=3 \mathrm{PD}$ and 3 controls) and of the PD prefrontal cortex from hemispheres matched and unmatched to the side of symptom predominance ( $n=5$ PD-matched and 5 PD-unmatched) was performed. Mass spectrometry analysis of prefrontal cortex samples ( $30 \mathrm{mg}$ tissue) was performed by the Integrated Mass Spectrometry Unit at Michigan State University. Briefly, protein lysate $(20 \mathrm{mg})$ was denatured using $25 \mathrm{mM}$ ammonium bicarbonate/80\% acetonitrile and incubated at $37^{\circ} \mathrm{C}$ for $3 \mathrm{~h}$. The samples were dried and reconstituted in $25 \mathrm{mM}$ ammonium bicarbonate/ $50 \%$ acetonitrile/trypsin solution and incubated overnight at $37{ }^{\circ} \mathrm{C}$. The resulting peptides were dried and reconstituted in $25 \mathrm{mM}$ ammonium bicarbonate/4\% acetonitrile. Samples were loaded on to a C18 column ( $2 \mathrm{~mm}$ particles, $25 \mathrm{~cm} \times 75 \mathrm{~mm}$ ID) and eluted using a 2-h acetonitrile gradient into a Q-Exactive HF-X mass spectrometer. Each sample was run in triplicate to account for technical variance. The mass spectra from each technical replicate were searched against the Uniprot human database using LFQ method in Proteome Discoverer (Version 2.2.0.388, 2017). The technical replicates from each biological sample were pooled and group comparisons (controls vs. PD, PD-matched vs PDunmatched) were performed using a non-nested test. Only proteins with abundances recorded in at least two samples per group were considered. Proteins with log fold change between groups exceeding \pm 0.2 were considered as altered. To identify protein changes in PD relevant to the lateralization of clinical symptoms, we first identified proteins differing between PD and controls, and then merged this protein list with those differing between matched and unmatched PD hemispheres. The resulting list of 345 genes corresponded to PDrelevant proteins with hemispheric asymmetry, and their interactions were visualized using STRING-db version 11 [104]. Pathway analysis of proteins involved in PD hemispheric asymmetry was done by g:Profiler [100] with networks determined by EnrichmentMap [102] and clustered by AutoAnnotate [103] in Cytoscape v3.7.1.

\section{Genetic variation associated with DNA methylation}

We determined the influence of cis-acting genetic variation on DNA methylation sites relevant to hemispheric asymmetry in PD. BS-SNPer [106] was used to identify SNPs proximal to the 815,367 cytosine sites profiled in the DNA methylation analysis of the cohort examining both hemispheres of the same PD and control individuals ( $n=31$ controls and 26 PD patients). SNPs were determined in each sample along with allele frequency by the BS-SNPer software [106] from bismark generated alignment .bam files. $\mathrm{CpG}$ and $\mathrm{CpH}$ sites were removed from the list of identified SNPs. SNPs with missing values in more than $50 \%$ samples were excluded. The resulting $\mathrm{SNPs}$ were assigned to $\mathrm{CpG} / \mathrm{CpH}$ sites if located within $\pm 500 \mathrm{~kb}$ of the cytosine site. An meQTL analysis was performed examining the effect of genotype on DNA methylation, adjusting for diagnosis, brain hemisphere, sex, age, postmortem interval, and neuronal subtype proportion. A Benjamini-Hochberg false discovery rate correction was performed, with FDR $q<0.05$ deemed significant. A $\chi^{2}$ test was used to compare the number of SNP-methylation associations for cytosines exhibiting hemispheric asymmetry in PD relative to all tested cytosines (background). Genes with SNPassociated epigenetic asymmetry in PD were determined by the gene targets of cytosine sites identified in the chromatin conformation data from human cortical neurons and excitatory neurons $[47,48]$, and the in silico cis-regulatory element prediction tool, as described above.

\section{Supplementary information}

Supplementary information accompanies this paper at https://doi.org/10. 1186/s13059-020-01960-1.

Additional file 1: Figure S1. Isolation of human prefrontal cortex neurons by flow cytometry. Figure S2. Analysis of cytosine site coverage and sample distributions showing high technical reproducibility in discovery cohort and replication cohort. Figure S3. Hemispheric asymmetry in controls and PD patients is replicated for $\mathrm{CpG}$ and $\mathrm{CpH}$ sites with $\geq 100 X$ read coverage. Figure S4. Genomic locations of differentially methylated neuronal enhancer/promoter cytosines 
associated with hemispheric asymmetry in controls and in PD patients. Figure S5. Genomic location of enrichment of DNA methylation changes involved in hemispheric asymmetry in human cortical neurons. Figure S6. Enhancers and promoters with DNA methylation changes in the symptom-dominant PD hemisphere have corresponding changes in the expression of their target genes. Figure S7. Hemispheric asymmetry in DNA methylation is prevalent in the human brain, as confirmed in the replication cohort. Figure S8. Epigenetic and transcriptional dysregulation is more prevalent in neurons of the symptom-dominant PD hemisphere than in neurons of the non-dominant hemisphere. Figure $\mathbf{5 9 .}$ Protein-protein interaction network of 345 proteins with altered abundance in PD relative to controls and that differed between the PD symptom-dominant and non-dominant hemisphere. Figure S10. In PD neurons, epigenetic changes with aging differ between the hemispheres matched and unmatched to symptom predominance. Figure S11. Pathways and genomic locations of DNA methylation changes relevant to hemispheric asymmetry in PD that were associated to genetic variation. Table S1. Inter-sample correlations for $\mathrm{CpG}$ and $\mathrm{CpH}$ in controls of the same hemisphere. Table S2. Software used for analysis.

Additional file 2: Demographic and clinical information for human samples.

Additional file 3: Primers

Additional file 4: Neuronal subtype deconvolution for prefrontal cortex neurons from hemispheres of controls and PD patients, discovery cohort.

Additional file 5: Differentially methylated cytosines involved in hemispheric asymmetry.

Additional file 6: Genes with epigenetic alterations at regulatory elements involved in hemispheric asymmetry.

Additional file 7: Transcript level changes in the PD hemisphere matched or unmatched to side of symptom predominance.

Additional file 8: Genes most associated with PD symptom lateralization and hemisphere changes with aging.

Additional file 9: Proteomic changes involved in hemispheric asymmetry and symptom lateralization in PD.

Additional file 10: Proteins exhibiting hemispheric asymmetry in PD that have corresponding gene transcripts and/or DNA methylation differences across PD hemispheres.

Additional file 11: Cis-acting genetic variation influences DNA methylation changes associated with hemispheric asymmetry in PD.

Additional file 12: Review history.

\section{Acknowledgements}

We thank the Van Andel Research Institute Flow Cytometry, Genomics, Bioinformatics and Biostatistics, and Pathology Cores. We thank Zachary Madaj in the Bioinformatics and Biostatistics Core at the Van Andel Research Institute for consulting on statistical approaches. We also thank the CAMH Sequencing Facility. We appreciate the manuscript reviews provided Dr. Art Petronis and Edward Oh. We thank the patient donors and families for the support of our research and the Parkinson's UK Brain Bank, the NIH NeuroBioBank, and the Michigan Brain Bank for the brain tissue provided.

\section{Peer review information}

Yixin Yao was the primary editor on this article and managed its editorial process and peer review in collaboration with the rest of the editorial team.

\section{Review history}

The review history is available as Additional file 12.

\section{Funding}

V.L. is supported by grants from the Department of Defense (W81XWH1810512), the National Institute of Neurological Disorders and Stroke (1R21NS112614-01 and 1R01NS113894-01A1), and a Gibby \& Friends vs. Parky Award.

\section{Availability of data and materials}

The datasets generated and analyzed during the current study are available in the NCBI Gene Expression Omnibus (GEO) database under GSE135037
[107]. The mass spectrometry proteomics data have been deposited to the ProteomeXchange Consortium via the PRIDE partner repository with the dataset identifiers PXD015079 [108] and PXD015239 [109]. Custom code for DNA methylation and RNA-seq analysis is available at https:/github.com/ lipeipei0611/PD_Asymmetry [110]. Software used in this study is detailed in Additional file 1: Table S2.

\section{Authors' contributions}

$\mathrm{PL}$ contributed to experimental design and performed all computational analyses. EE performed the neuronal nuclei isolations and contributed to bisulfite padlock probe library preparation. LM contributed to the RNA-seq analysis. MS, JL, and IV performed the proteomics analysis. VL was involved with study design and overseeing the experiments. The manuscript was written by $\mathrm{VL}, \mathrm{SL}$, and $\mathrm{PL}$, and all authors commented on the manuscript. The authors read and approved the final manuscript.

\section{Ethics approval and consent to participate}

Human prefrontal cortex tissue for this study was obtained from the Parkinson's UK Brain Bank, NIH NeuroBioBank, and Michigan Brain Bank, with approval from the ethics committee of the Van Andel Research Institute (IRB \#15025) and conformed with the Declaration of Helsinki.

\section{Consent for publication}

Not applicable.

\section{Competing interests}

The authors declare that they have no competing interests.

\section{Author details}

${ }^{1}$ Center for Neurodegenerative Science, Van Andel Institute, Grand Rapids, MI 49503, USA. 'Department of Translational Neuroscience, College of Human Medicine, Michigan State University, Grand Rapids, MI 49503, USA. ${ }^{3}$ Integrated Mass Spectrometry Unit, College of Human Medicine, Michigan State University, Grand Rapids, MI 49503, USA. ${ }^{4}$ Division of Psychiatry and Behavioral Medicine, College of Human Medicine, Michigan State University, Grand Rapids, Ml 49503, USA.

Received: 11 October 2019 Accepted: 13 February 2020

Published online: 09 March 2020

\section{References}

1. Djaldetti R, Ziv I, Melamed E. The mystery of motor asymmetry in Parkinson's disease. Lancet Neurol. 2006;5:796-802.

2. Barrett MJ, Wylie SA, Harrison MB, Wooten GF. Handedness and motor symptom asymmetry in Parkinson's disease. J Neurol Neurosurg Psychiatry. 2011;82:1122-4.

3. Pagano G, Ferrara N, Brooks DJ, Pavese N. Age at onset and Parkinson disease phenotype. Neurology. 2016;86:1400-7.

4. Wang J, Yang QX, Sun X, Vesek J, Mosher Z, Vasavada M, Chu J, Kanekar S, Shivkumar V, Venkiteswaran K, Subramanian T. MRl evaluation of asymmetry of nigrostriatal damage in the early stage of early-onset Parkinson's disease. Parkinsonism Relat Disord. 2015:21:590-6.

5. Claassen DO, McDonell KE, Donahue M, Rawal S, Wylie SA, Neimat JS, Kang H, Hedera P, Zald D, Landman B, et al. Cortical asymmetry in Parkinson's disease: early susceptibility of the left hemisphere. Brain Behav. 2016;6: e00573.

6. Sixel-Döring F, Liepe K, Mollenhauer B, Trautmann E, Trenkwalder C. The role of 123I-FP-CIT-SPECT in the differential diagnosis of Parkinson and tremor syndromes: a critical assessment of 125 cases. J Neurol. 2011;258: 2147-54.

7. Riederer $\mathrm{P}$, Sian-Hülsmann J. The significance of neuronal lateralisation in Parkinson's disease. J Neural Transm (Vienna). 2012;119:953-62.

8. Bohnen NI, Albin RL, Koeppe RA, Wernette KA, Kilbourn MR, Minoshima S, Frey KA. Positron emission tomography of monoaminergic vesicular binding in aging and Parkinson disease. J Cereb Blood Flow Metab. 2006;26:1198212.

9. Iranzo A, Stefani A, Serradell M, Marti MJ, Lomena F, Mahlknecht P, Stockner H, Gaig C, Fernandez-Arcos A, Poewe W, et al. Characterization of patients with longstanding idiopathic REM sleep behavior disorder. Neurology. 2017; 89:242-8. 
10. Djaldetti R, Lorberboym M, Karmon Y, Treves TA, Ziv I, Melamed E. Residual striatal dopaminergic nerve terminals in very long-standing Parkinson's disease: a single photon emission computed tomography imaging study. Mov Disord. 2011:26:327-30.

11. Poewe W, Seppi K, Tanner CM, Halliday GM, Brundin P, Volkmann J, Schrag AE, Lang AE. Parkinson disease. Nat Rev Dis Primers. 2017;3:17013.

12. Lawton M, Ben-Shlomo Y, May MT, Baig F, Barber TR, Klein JC, Swallow DMA, Malek N, Grosset KA, Bajaj N, et al. Developing and validating Parkinson's disease subtypes and their motor and cognitive progression. J Neurol Neurosurg Psychiatry. 2018;89:1279-87.

13. Marinus J, van Hilten JJ. The significance of motor (a)symmetry in Parkinson's disease. Mov Disord. 2015;30:379-85.

14. Baumann CR, Held U, Valko PO, Wienecke M, Waldvogel D. Body side and predominant motor features at the onset of Parkinson's disease are linked to motor and nonmotor progression. Mov Disord. 2014;29:207-13.

15. Huang P, Tan YY, Liu DQ, Herzallah MM, Lapidow E, Wang Y, Zang YF, Gluck MA, Chen SD. Motor-symptom laterality affects acquisition in Parkinson's disease: a cognitive and functional magnetic resonance imaging study. Mov Disord. 2017;32:1047-55.

16. Rodríguez-Violante M, Cervantes-Arriaga A, Villar-Velarde A, Corona T. Relationship between the type and side of motor symptoms with the prevalence of non-motor symptoms in Parkinson's disease. Neurologia. 2011;26:319-24.

17. Lee EY, Sen S, Eslinger PJ, Wagner D, Kong L, Lewis MM, Du G, Huang X. Side of motor onset is associated with hemisphere-specific memory decline and lateralized gray matter loss in Parkinson's disease. Parkinsonism Relat Disord. 2015;21:465-70.

18. Karádi K, Lucza T, Aschermann Z, Komoly S, Deli G, Bosnyák E, Acs P, Horváth $\mathrm{R}$, Janszky J, Kovács N. Visuospatial impairment in Parkinson's disease: the role of laterality. Laterality. 2015;20:112-27.

19. Cubo E, Martín PM, Martin-Gonzalez JA, Rodríguez-Blázquez C, Kulisevsky J, Members EG. Motor laterality asymmetry and nonmotor symptoms in Parkinson's disease. Mov Disord. 2010;25:70-5.

20. Lister R, Mukamel EA, Nery JR, Urich M, Puddifoot CA, Johnson ND, Lucero J, Huang Y, Dwork AJ, Schultz MD, et al. Global epigenomic reconfiguration during mammalian brain development. Science. 2013;341:1237905.

21. Guo JU, Su Y, Shin JH, Shin J, Li H, Xie B, Zhong C, Hu S, Le T, Fan G, et al. Distribution, recognition and regulation of non-CpG methylation in the adult mammalian brain. Nat Neurosci. 2014;17:215-22.

22. Davies MN, Volta M, Pidsley R, Lunnon K, Dixit A, Lovestone S, Coarfa C, Harris RA, Milosavljevic A, Troakes C, et al. Functional annotation of the human brain methylome identifies tissue-specific epigenetic variation across brain and blood. Genome Biol. 2012;13:R43.

23. Rizzardi LF, Hickey PF, Rodriguez DiBlasi V, Tryggvadóttir R, Callahan CM, Idrizi A, Hansen KD, Feinberg AP. Neuronal brain-region-specific DNA methylation and chromatin accessibility are associated with neuropsychiatric trait heritability. Nat Neurosci. 2019;22:307-16.

24. Zhang TY, Keown CL, Wen X, Li J, Vousden DA, Anacker C, Bhattacharyya U, Ryan R, Diorio J, O'Toole N, et al. Environmental enrichment increases transcriptional and epigenetic differentiation between mouse dorsal and ventral dentate gyrus. Nat Commun. 2018;9:298.

25. Ocklenburg S, Schmitz J, Moinfar Z, Moser D, Klose R, Lor S, Kunz G, Tegenthoff $M$, Faustmann $P$, Francks $C$, et al. Epigenetic regulation of lateralized fetal spinal gene expression underlies hemispheric asymmetries. Elife. 2017;6.

26. de Kovel CGF, Lisgo SN, Fisher SE, Francks C. Subtle left-right asymmetry of gene expression profiles in embryonic and foetal human brains. Sci Rep. 2018:8:12606.

27. Schmitz J, Metz GAS, Gunturkun O, Ocklenburg S. Beyond the genometowards an epigenetic understanding of handedness ontogenesis. Prog Neurobiol. 2017;159:69-89.

28. Krebs LT, Iwai N, Nonaka S, Welsh IC, Lan Y, Jiang R, Saijoh Y, O'Brien TP, Hamada $\mathrm{H}$, Gridley T. Notch signaling regulates left-right asymmetry determination by inducing nodal expression. Genes Dev. 2003;17:1207-12.

29. Welsh IC, Kwak H, Chen FL, Werner M, Shopland LS, Danko CG, Lis JT, Zhang M, Martin JF, Kurpios NA. Chromatin architecture of the Pitx2 locus requires CTCF- and Pitx2-dependent asymmetry that mirrors embryonic gut laterality. Cell Rep. 2015;13:337-49.

30. Wang L, Liu Z, Lin H, Ma D, Tao Q, Liu F. Epigenetic regulation of left-right asymmetry by DNA methylation. EMBO J. 2017;36:2987-97.

31. Halder R, Hennion M, Vidal RO, Shomroni O, Rahman RU, Rajput A, Centeno TP, van Bebber F, Capece V, Garcia Vizcaino JC, et al. DNA methylation changes in plasticity genes accompany the formation and maintenance of memory. Nat Neurosci. 2016;19:102-10.

32. Li P, Marshall L, Oh G, Jakubowski JL, Groot D, He Y, Wang T, Petronis A, Labrie $V$. Epigenetic dysregulation of enhancers in neurons is associated with Alzheimer's disease pathology and cognitive symptoms. Nat Commun. 2019;10:2246.

33. Hwang JY, Aromolaran KA, Zukin RS. The emerging field of epigenetics in neurodegeneration and neuroprotection. Nat Rev Neurosci. 2017;18:347-61.

34. Dashtipour K, Tafreshi A, Adler C, Beach T, Chen X, Serrano G, Tashiro S, Wang C. Hypermethylation of Synphilin-1, alpha-Synuclein-interacting protein (SNCAIP) gene in the cerebral cortex of patients with sporadic Parkinson's disease. Brain Sci. 2017;7(7):74.

35. Masliah E, Dumaop W, Galasko D, Desplats P. Distinctive patterns of DNA methylation associated with Parkinson disease: identification of concordant epigenetic changes in brain and peripheral blood leukocytes. Epigenetics. 2013;8:1030-8.

36. Young JI, Sivasankaran SK, Wang L, Ali A, Mehta A, Davis DA, Dykxhoorn DM, Petito CK, Beecham GW, Martin ER, et al. Genome-wide brain DNA methylation analysis suggests epigenetic reprogramming in Parkinson disease. Neurol Genet. 2019;5:e342.

37. Consortium. IPsDG, Consortium. WTCC: A two-stage meta-analysis identifies several new loci for Parkinson's disease. PLoS Genet. 2011;7:e1002142.

38. Pihlstrom L, Berge V, Rengmark A, Toft M. Parkinson's disease correlates with promoter methylation in the alpha-synuclein gene. Mov Disord. 2015;30:577-80.

39. Jowaed A, Schmitt I, Kaut O, Wullner U. Methylation regulates alphasynuclein expression and is decreased in Parkinson's disease patients' brains. J Neurosci. 2010;30:6355-9.

40. Grundemann J, Schlaudraff F, Haeckel O, Liss B. Elevated alpha-synuclein mRNA levels in individual UV-laser-microdissected dopaminergic substantia nigra neurons in idiopathic Parkinson's disease. Nucleic Acids Res. 2008:36:e38.

41. Shahmoradian SH, Lewis AJ, Genoud C, Hench J, Moors TE, Navarro PP, Castano-Diez D, Schweighauser G, Graff-Meyer A, Goldie KN, et al. Lewy pathology in Parkinson's disease consists of crowded organelles and lipid membranes. Nat Neurosci. 2019:22:1099-109.

42. Horvath S, Zhang Y, Langfelder P, Kahn RS, Boks MP, van Eijk K, van den Berg LH, Ophoff RA. Aging effects on DNA methylation modules in human brain and blood tissue. Genome Biol. 2012;13:R97.

43. van Dongen J, Nivard MG, Willemsen G, Hottenga JJ, Helmer Q, Dolan CV, Ehli EA, Davies GE, van Iterson M, Breeze CE, et al. Genetic and environmental influences interact with age and sex in shaping the human methylome. Nat Commun. 2016;7:11115.

44. Lu AT, Hannon E, Levine ME, Crimmins EM, Lunnon K, Mill J, Geschwind DH, Horvath S. Genetic architecture of epigenetic and neuronal ageing rates in human brain regions. Nat Commun. 2017;8:15353.

45. Horvath S, Ritz BR. Increased epigenetic age and granulocyte counts in the blood of Parkinson's disease patients. Aging (Albany NY). 2015;7:1130-42.

46. Pai S, Li P, Killinger B, Marshall L, Jia P, Liao J, Petronis A, Szabó PE, Labrie V. Differential methylation of enhancer at IGF2 is associated with abnormal dopamine synthesis in major psychosis. Nat Commun. 2019;10:2046.

47. Nott A, Holtman IR, Coufal NG, Schlachetzki JCM, Yu M, Hu R, Han CZ, Pena M, Xiao J, Wu Y, et al. Brain cell type-specific enhancer-promoter interactome maps and disease-risk association. Science. 2019;366:1134-9.

48. Song M, Yang X, Ren X, Maliskova L, Li B, Jones IR, Wang C, Jacob F, Wu K, Traglia $M$, et al. Mapping cis-regulatory chromatin contacts in neural cells links neuropsychiatric disorder risk variants to target genes. Nat Genet. 2019; 51:1252-62.

49. McLean CY, Bristor D, Hiller M, Clarke SL, Schaar BT, Lowe CB, Wenger AM, Bejerano G. GREAT improves functional interpretation of cis-regulatory regions. Nat Biotechnol. 2010;28:495-501.

50. Chang D, Nalls MA, Hallgrímsdóttir IB, Hunkapiller J, van der Brug M, Cai F, Kerchner GA, Ayalon G, Bingol B, Sheng M, et al. A meta-analysis of genome-wide association studies identifies 17 new Parkinson's disease risk loci. Nat Genet. 2017;49:1511-6.

51. Risso D, Ngai J, Speed TP, Dudoit S. Normalization of RNA-seq data using factor analysis of control genes or samples. Nat Biotechnol. 2014;32:896-902.

52. Pan $P$, Zhang $Y$, Liu Y, Zhang H, Guan D, Xu Y. Abnormalities of regional brain function in Parkinson's disease: a meta-analysis of resting state functional magnetic resonance imaging studies. Sci Rep. 2017:7:40469.

53. Jubault T, Gagnon JF, Karama S, Ptito A, Lafontaine AL, Evans AC, Monchi O. Patterns of cortical thickness and surface area in early Parkinson's disease. Neuroimage. 2011;55:462-7. 
54. Scherfler C, Seppi K, Mair KJ, Donnemiller E, Virgolini I, Wenning GK, Poewe W. Left hemispheric predominance of nigrostriatal dysfunction in Parkinson's disease. Brain. 2012;135:3348-54.

55. Yau Y, Zeighami Y, Baker TE, Larcher K, Vainik U, Dadar M, Fonov VS, Hagmann P, Griffa A, Misic B, et al. Network connectivity determines cortical thinning in early Parkinson's disease progression. Nat Commun. 2018;9:12.

56. van Woerden GM, Hoebeek FE, Gao Z, Nagaraja RY, Hoogenraad CC, Kushner SA, Hansel C, De Zeeuw Cl. Elgersma Y: betaCaMKIl controls the direction of plasticity at parallel fiber-Purkinje cell synapses. Nat Neurosci. 2009:12:823-5.

57. Kool MJ, van de Bree JE, Bodde HE, Elgersma Y, van Woerden GM. The molecular, temporal and region-specific requirements of the beta isoform of calcium/calmodulin-dependent protein kinase type 2 (CAMK2B) in mouse locomotion. Sci Rep. 2016;6:26989.

58. Sando R, Gounko N, Pieraut S, Liao L, Yates J, Maximov A. HDAC4 governs a transcriptional program essential for synaptic plasticity and memory. Cell. 2012;151:821-34.

59. Ko HG, Choi JH, Park DI, Kang SJ, Lim CS, Sim SE, Shim J, Kim Jl, Kim S, Choi $\mathrm{TH}$, et al. Rapid turnover of cortical NCAM1 regulates synaptic reorganization after peripheral nerve injury. Cell Rep. 2018;22:748-59.

60. Miners JS, Love S. Endothelin-converting enzymes degrade a-synuclein and are reduced in dementia with Lewy bodies. J Neurochem. 2017;141:275-86.

61. Sauer K, Cooke MP. Regulation of immune cell development through soluble inositol-1,3,4,5-tetrakisphosphate. Nat Rev Immunol. 2010;10:257-71.

62. Kitagawa Y, Ohkura N, Kidani Y, Vandenbon A, Hirota K, Kawakami R, Yasuda K, Motooka D, Nakamura S, Kondo M, et al. Guidance of regulatory T cell development by Satb1-dependent super-enhancer establishment. Nat Immunol. 2017;18:173-83.

63. Lorenzo DN, Badea A, Davis J, Hostettler J, He J, Zhong G, Zhuang X, Bennett V. A PIK3C3-ankyrin-B-dynactin pathway promotes axonal growth and multiorganelle transport. J Cell Biol. 2014;207:735-52.

64. Hell JW. CaMKIl: claiming center stage in postsynaptic function and organization. Neuron. 2014;81:249-65.

65. Price AJ, Collado-Torres L, Ivanov NA, Xia W, Burke EE, Shin JH, Tao R, Ma L, Jia $Y$, Hyde TM, et al. Divergent neuronal DNA methylation patterns across human cortical development reveal critical periods and a unique role of CpH methylation. Genome Biol. 2019;20:196.

66. Stroud H, Su SC, Hrvatin S, Greben AW, Renthal W, Boxer LD, Nagy MA, Hochbaum DR, Kinde B, Gabel HW, Greenberg ME. Early-life gene expression in neurons modulates lasting epigenetic states. Cell. 2017;171: 1151-64. e1116.

67. van der Hoorn A, Burger $\mathrm{H}$, Leenders KL, de Jong BM. Handedness correlates with the dominant Parkinson side: a systematic review and metaanalysis. Mov Disord. 2012;27:206-10.

68. Stochl J, Hagtvet KA, Brozova H, Klempir J, Roth J, Ruzicka E. Handedness does not predict side of onset of motor symptoms in Parkinson's disease. Mov Disord. 2009:24:1836-9.

69. van Rooden SM, Visser M, Verbaan D, Marinus J. Handedness associated to side of onset of Parkinson's disease? Parkinsonism Relat Disord. 2009;15: $546-7$.

70. Xiao MF, Xu JC, Tereshchenko Y, Novak D, Schachner M, Kleene R. Neural cell adhesion molecule modulates dopaminergic signaling and behavior by regulating dopamine D2 receptor internalization. J Neurosci. 2009;29:14752-63.

71. Van Acker HH, Capsomidis A, Smits EL, Van Tendeloo VF. CD56 in the immune system: more than a marker for cytotoxicity? Front Immunol. 2017:8:892

72. Henderson MX, Cornblath EJ, Darwich A, Zhang B, Brown H, Gathagan RJ, Sandler RM, Bassett DS, Trojanowski JQ, Lee VMY. Spread of alpha-synuclein pathology through the brain connectome is modulated by selective vulnerability and predicted by network analysis. Nat Neurosci. 2019;22:1248-57.

73. Zheng YQ, Zhang Y, Yau Y, Zeighami Y, Larcher K, Misic B, Dagher A. Local vulnerability and global connectivity jointly shape neurodegenerative disease propagation. PLoS Biol. 2019;17:e3000495.

74. Cabeza R. Hemispheric asymmetry reduction in older adults: the HAROLD model. Psychol Aging. 2002;17:85-100.

75. Dolcos F, Rice HJ, Cabeza R. Hemispheric asymmetry and aging: right hemisphere decline or asymmetry reduction. Neurosci Biobehav Rev. 2002; 26:819-25.

76. Arnò B, Grassivaro F, Rossi C, Bergamaschi A, Castiglioni V, Furlan R, Greter M, Favaro R, Comi G, Becher B, et al. Neural progenitor cells orchestrate microglia migration and positioning into the developing cortex. Nat Commun. 2014;5:5611.
77. Nimmerjahn A, Kirchhoff F, Helmchen F. Resting microglial cells are highly dynamic surveillants of brain parenchyma in vivo. Science. 2005;308:1314-8.

78. Masuda T, Sankowski R, Staszewski O, Böttcher C, Amann L, Sagar SC, Nessler S, Kunz P, van Loo G, et al. Spatial and temporal heterogeneity of mouse and human microglia at single-cell resolution. Nature. 2019;566:388-92.

79. Steiner J, Mawrin C, Ziegeler A, Bielau H, Ullrich O, Bernstein HG, Bogerts B. Distribution of HLA-DR-positive microglia in schizophrenia reflects impaired cerebral lateralization. Acta Neuropathol. 2006;112:305-16.

80. Zhan Y, Paolicelli RC, Sforazzini F, Weinhard L, Bolasco G, Pagani F, Vyssotski AL, Bifone A, Gozzi A, Ragozzino D, Gross CT. Deficient neuron-microglia signaling results in impaired functional brain connectivity and social behavior. Nat Neurosci. 2014;17:400-6.

81. Paolicelli RC, Bolasco G, Pagani F, Maggi L, Scianni M, Panzanelli P, Giustetto $M$, Ferreira TA, Guiducci E, Dumas L, et al. Synaptic pruning by microglia is necessary for normal brain development. Science. 2011;333:1456-8.

82. Narayanan NS, Rodnitzky RL, UC EY. Prefrontal dopamine signaling and cognitive symptoms of Parkinson's disease. Rev Neurosci. 2013;24:267-78.

83. Braak H, Del Tredici K, Rüb U, de Vos RA, Jansen Steur EN, Braak E. Staging of brain pathology related to sporadic Parkinson's disease. Neurobiol Aging. 2003;24:197-211.

84. Weintraub D, Doshi J, Koka D, Davatzikos C, Siderowf AD, Duda JE, Wolk DA Moberg PJ, Xie SX, Clark CM. Neurodegeneration across stages of cognitive decline in Parkinson disease. Arch Neurol. 2011;68:1562-8.

85. Labrie V, Buske OJ, Oh E, Jeremian R, Ptak C, Gasiūnas G, Maleckas A, Petereit $\mathrm{R}$, Žvirbliene A, Adamonis $\mathrm{K}$, et al. Lactase nonpersistence is directed by DNA-variation-dependent epigenetic aging. Nat Struct Mol Biol. 2016;23:566-73.

86. He Y, Wang T. EpiCompare: an online tool to define and explore genomic regions with tissue or cell type-specific epigenomic features. Bioinformatics. 2017:33:3268-75.

87. Ernst J, Kellis M. ChromHMM: automating chromatin-state discovery and characterization. Nat Methods. 2012;9:215-6.

88. Kundaje A, Meuleman W, Ernst J, Bilenky M, Yen A, Heravi-Moussavi A, Kheradpour P, Zhang Z, Wang J, Ziller MJ, et al. Integrative analysis of 111 reference human epigenomes. Nature. 2015;518:317-30.

89. Diep D, Plongthongkum N, Gore A, Fung HL, Shoemaker R, Zhang K. Library-free methylation sequencing with bisulfite padlock probes. Nat Methods. 2012;9:270-2.

90. Krueger F, Andrews SR. Bismark: a flexible aligner and methylation caller for bisulfite-Seq applications. Bioinformatics. 2011;27:1571-2.

91. Auton A, Brooks LD, Durbin RM, Garrison EP, Kang HM, Korbel JO, Marchini JL, McCarthy S, McVean GA, Abecasis GR, Consortium GP. A global reference for human genetic variation. Nature. 2015;526:68-74.

92. Luo C, Keown CL, Kurihara L, Zhou J, He Y, Li J, Castanon R, Lucero J, Nery $J R$, Sandoval JP, et al. Single-cell methylomes identify neuronal subtypes and regulatory elements in mammalian cortex. Science. 2017;357:600-4

93. Newman AM, Liu CL, Green MR, Gentles AJ, Feng W, Xu Y, Hoang CD, Diehn M, Alizadeh AA. Robust enumeration of cell subsets from tissue expression profiles. Nat Methods. 2015;12:453-7.

94. Ritchie ME, Phipson B, Wu D, Hu Y, Law CW, Shi W. Smyth GK: limma powers differential expression analyses for RNA-sequencing and microarray studies. Nucleic Acids Res. 2015;43:e47.

95. Du P, Kibbe WA, Lin SM. lumi: a pipeline for processing Illumina microarray. Bioinformatics. 2008;24:1547-8.

96. Dobin A, Davis CA, Schlesinger F, Drenkow J, Zaleski C, Jha S, Batut P, Chaisson M, Gingeras TR. STAR: ultrafast universal RNA-seq aligner. Bioinformatics. 2013;29:15-21.

97. Robinson MD, McCarthy DJ, Smyth GK. edgeR: a bioconductor package for differential expression analysis of digital gene expression data. Bioinformatics. 2010;26:139-40.

98. Yu Q, He Z. Comprehensive investigation of temporal and autism-associated cell type composition-dependent and independent gene expression changes in human brains. Sci Rep. 2017;7:4121.

99. Darmanis S, Sloan SA, Zhang Y, Enge M, Caneda C, Shuer LM, Hayden Gephart MG, Barres BA, Quake SR. A survey of human brain transcriptome diversity at the single cell level. Proc Natl Acad Sci U S A. 2015;112:7285-90.

100. Raudvere U, Kolberg L, Kuzmin I, Arak T, Adler P, Peterson H, Vilo J. g: Profiler: a web server for functional enrichment analysis and conversions of gene lists (2019 update). Nucleic Acids Res. 2019;47:W191-8.

101. Subramanian A, Tamayo P, Mootha VK, Mukherjee S, Ebert BL, Gillette MA, Paulovich A, Pomeroy SL, Golub TR, Lander ES, Mesirov JP. Gene set 
enrichment analysis: a knowledge-based approach for interpreting genomewide expression profiles. Proc Natl Acad Sci U S A. 2005;102:15545-50.

102. Merico D, Isserlin R, Stueker O, Emili A, Bader GD. Enrichment map: a network-based method for gene-set enrichment visualization and interpretation. PLoS One. 2010;5:e13984.

103. Kucera M, Isserlin R, Arkhangorodsky A, Bader GD. AutoAnnotate: A Cytoscape app for summarizing networks with semantic annotations. F1000Res. 2016;5:1717.

104. Szklarczyk D, Morris JH, Cook H, Kuhn M, Wyder S, Simonovic M, Santos A, Doncheva NT, Roth A, Bork P, et al. The STRING database in 2017: qualitycontrolled protein-protein association networks, made broadly accessible. Nucleic Acids Res. 2017;45:D362-8.

105. Kolde R, Laur S, Adler P, Vilo J. Robust rank aggregation for gene list integration and meta-analysis. Bioinformatics. 2012;28:573-80.

106. Gao S, Zou D, Mao L, Liu H, Song P, Chen Y, Zhao S, Gao C, Li X, Gao Z, et al. BS-SNPer: SNP calling in bisulfite-seq data. Bioinformatics. 2015;31: 4006-8.

107. Li P, Ensink E, Lang S, Marshall L, Schilthuis M, Lamp J, Vega I, Labrie V. Hemispheric asymmetry in the human brain and in Parkinson's disease is linked to divergent epigenetic patterns in neurons. Gene Expression Omnibus. https://www.ncbi.nlm.nih.gov/geo/query/acc.cgi?acc=GSE135037. Accessed 12 Feb 2020

108. Gordevicius J, Li P, Marshall L, Killinger B, Lang S, Ensink E, Kuhn NC, Cui W, Maroof N, Lauria R, et al. Epigenetic inactivation of the autophagy-lysosomal system in the Parkinson's disease appendix. PRIDE Archive. https://www.ebi.ac. uk/pride/archive/projects/PXD015079. Accessed 17 Feb 2020

109. Li P, Ensink E, Lang S, Marshall L, Schilthuis M, Lamp J, Vega I, Labrie V. Hemispheric asymmetry in the human brain and in Parkinson's disease is linked to divergent epigenetic patterns in neurons. PRIDE Archive: https:// www.ebi.ac.uk/pride/archive/projects/PXD015239. Accessed 17 Feb 2020.

110. Li P, Ensink E, Lang S, Marshall L, Schilthuis M, Lamp J, Vega I, Labrie V. Hemispheric asymmetry in the human brain and in Parkinson's disease is linked to divergent epigenetic patterns in neurons. GitHub: https://github. com/lipeipei0611/PD_Asymmetry. Accessed 12 Feb 2020.

\section{Publisher's Note}

Springer Nature remains neutral with regard to jurisdictional claims in published maps and institutional affiliations.

Ready to submit your research? Choose BMC and benefit from:

- fast, convenient online submission

- thorough peer review by experienced researchers in your field

- rapid publication on acceptance

- support for research data, including large and complex data types

- gold Open Access which fosters wider collaboration and increased citations

- maximum visibility for your research: over $100 \mathrm{M}$ website views per year

At $\mathrm{BMC}$, research is always in progress.

Learn more biomedcentral.com/submissions 